\title{
EVALUATION OF SOIL RADIOACTIVITY DATA FROM THE NEVADA TEST SITE
}

\author{
Prepared for \\ DOE Nevada Operations Office \\ Las Vegas, Nevada \\ Prepared by \\ IT CORPORATION \\ 4330 South Valley View Boulevard, Suite 114 \\ Las Vegas, Nevada 89103 \\ and \\ Desert Research Institute \\ 755 East Flamingo Road \\ Las Vegas, Nevada
}

March 1995

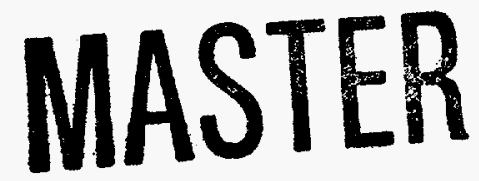




\section{DISCLAIMER}

This report was prepared as an account of work sponsored by an agency of the United States Government. Neither the United States Government nor any agency thereof, nor any of their employees, make any warranty, express or implied, or assumes any legal liability or responsibility for the accuracy, completeness, or usefulness of any information, apparatus, product, or process disclosed, or represents that its use would not infringe privately owned rights. Reference herein to any specific commercial product, process, or service by trade name, trademark, manufacturer, or otherwise does not necessarily constitute or imply its endorsement, recommendation, or favoring by the United States Government or any agency thereof. The views and opinions of authors expressed herein do not necessarily state or reflect those of the United States Government or any agency thereof. 


\section{DISCLAIMER}

Portions of this document may be illegible in electronic image products. Images are produced from the best available original document. 


\section{Table of Contents}

List of Figures $\ldots \ldots \ldots \ldots \ldots \ldots \ldots \ldots \ldots \ldots \ldots \ldots \ldots \ldots$

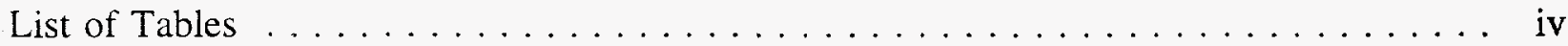

List of Acronyms and Abbreviations $\ldots \ldots \ldots \ldots \ldots \ldots \ldots$

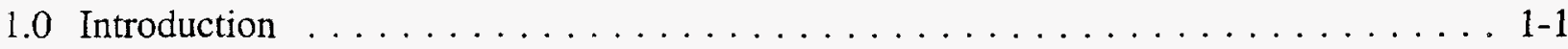

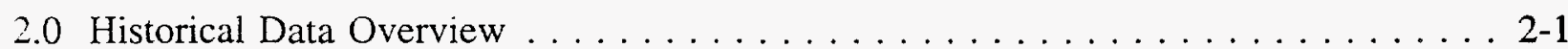

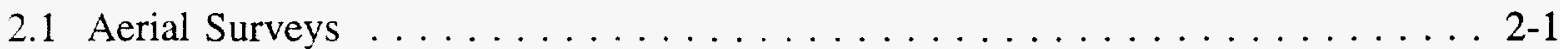

2.2 Nevada Test Site Radiological Assessment Project . . . . . . . . . . . . . 2-1

2.3 Nevada Applied Ecology Group . . . . . . . . . . . . . . . . . . . . 2-3

2.4 Radionuclide Inventory and Distribution Program . . . . . . . . . . . 2-4

3.0 The Nevada Applied Ecology Group Program . . . . . . . . . . . . . . 3-1

3.1 Data Collection . . . . . . . . . . . . . . . . . . . 3-3

3.1.1 Distribution and Inventory Element . . . . . . . . . . 3-3

3.1.2 Plutonium Inventory and Distribution Program Intensive Soil Sampling Phase . . . . . . . . . . . . . . . . . . . . . . . . . 3-4

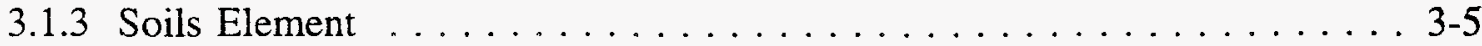

3.2 Sampling Techniques and Field Analytical Methods for Plutonium Inventory and Distribution Program and Soils Elements . . . . . . . . . . . . . 3-7

3.2.1 Surface Sampling . . . . . . . . . . . . . . . . . . 3-7

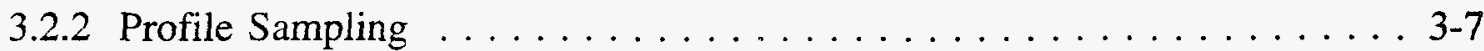

3.2 .3 Decontamination ....................... 3-8

3.2 .4 Number of Samples . . . . . . . . . . . . . . . . . . 3-8

3.2.5 Instrumentation and Source Checks $\ldots \ldots \ldots \ldots \ldots \ldots \ldots \ldots$

3.2.6 Sample Preparation $\ldots \ldots \ldots \ldots \ldots \ldots \ldots \ldots \ldots \ldots . \ldots \ldots$

3.3 Analytical Methods . . . . . . . . . . . . . . . . . . . . . . 3-10

3.3.1 Plutonium Inventory and Distribution Program Samples . . . . . . . . 3-10

3.3.2 Soils Element Samples . . . . . . . . . . . . . . . . . . 3-11

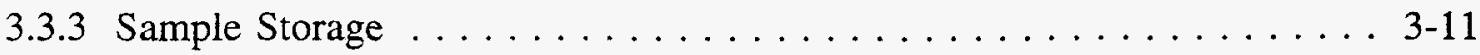


3.4 Records and Custody . . . . . . . . . . . . . . . . 3-11

3.4.1 Documentation $3-11$

3.4.2 Sample Custody 3-12

3.5 Quality Assurance Program $\ldots \ldots \ldots \ldots \ldots \ldots \ldots \ldots \ldots . \ldots \ldots$. . . . . . . . . .

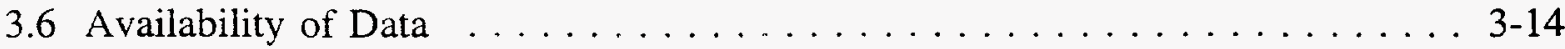

4.0 Radionuclide Inventory and Distribution Program $\ldots \ldots \ldots \ldots \ldots \ldots .4 .1$

4.1 In Situ Radiological Surveys $\ldots \ldots \ldots \ldots \ldots \ldots \ldots \ldots \ldots \ldots \ldots \ldots \ldots \ldots \ldots .2$

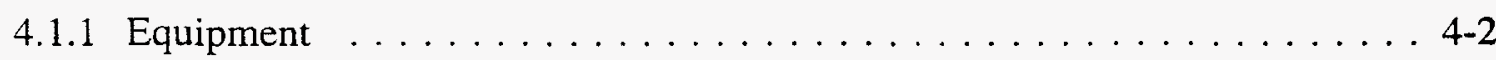

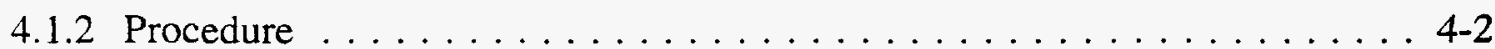

4.1.3 Determining Measurement Location . . . . . . . . . . . . . . . . 4-3

4.1.4 Spectral Processing and Analysis . . . . . . . . . . . . . . 4-4

4.1 .5 Conversion Factors $\ldots \ldots \ldots \ldots \ldots \ldots \ldots \ldots \ldots . . \ldots \ldots$

4.1 .6 Detection Limits $\ldots \ldots \ldots \ldots \ldots \ldots \ldots \ldots \ldots \ldots \ldots \ldots .6 \ldots \ldots$

4.1 .7 Records and Custody $\ldots \ldots \ldots \ldots \ldots \ldots \ldots \ldots \ldots \ldots \ldots .6 \ldots$

4.1 .8 Data Handling $\ldots \ldots \ldots \ldots \ldots \ldots \ldots \ldots \ldots \ldots . . \ldots \ldots . . \ldots \ldots$

4.1 .9 Quality Control $\ldots \ldots \ldots \ldots \ldots \ldots \ldots \ldots \ldots \ldots \ldots \ldots .7$

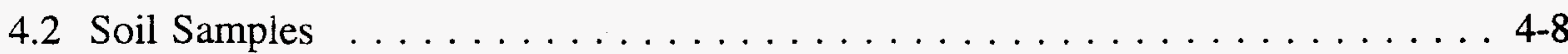

4.2.1 Sample Collection $\ldots \ldots \ldots \ldots \ldots \ldots \ldots \ldots \ldots \ldots$. $\ldots \ldots \ldots$

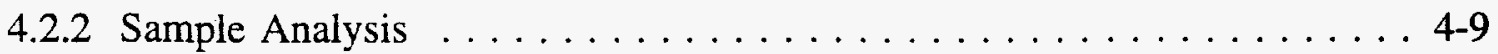

4.3 Inverse Relaxation Lengths and Radionuclide Ratios . . . . . . . . . . . . 4-10

4.4 Quality Assurance $\ldots \ldots \ldots \ldots \ldots \ldots \ldots \ldots \ldots \ldots \ldots \ldots . \ldots \ldots . \ldots \ldots$

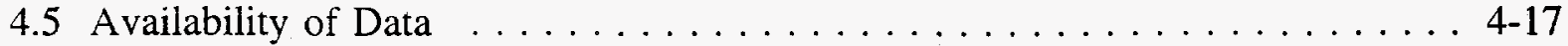

5.0 Discussion of Historical Data Useability $\ldots \ldots \ldots \ldots \ldots \ldots \ldots \ldots \ldots$ 5-1

5.1 Discrete Soil Samples . . . . . . . . . . . . . . . . . . 5-1

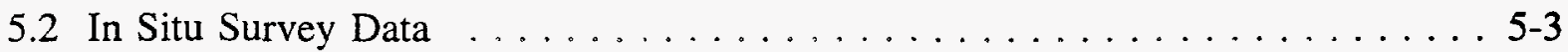

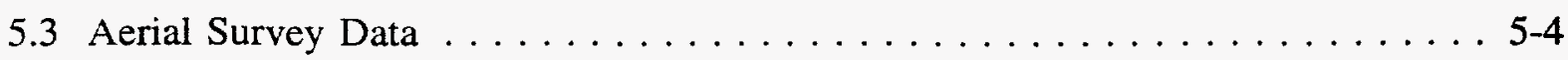

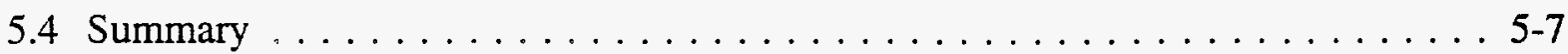

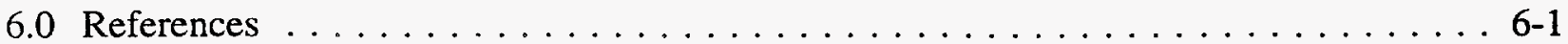




\section{List of Figures}

Number

Title

Page

1-1 Potential Plutonium-Contaminated Soil Sites at Nuclear Test Locations . . . . . . 1-2

2-1 Nevada Test Site EG\&G Aerial Survey Summary . . . . . . . . . . . . . 2-2

2-2 Nevada Test Site Nevada Applied Ecology Group Soil Sample Summary . . . . 2-5

2-3 Nevada Test Site Radionuclide Inventory and Distribution Program

Soil Sample Summary . . . . . . . . . . . . . . . . . . . . . . . . . . 2-6

2-4 Nevada Test Site Radionuclide Inventory and Distribution Program In Situ

Measurement Location Summary . . . . . . . . . . . . . . . . . . . . . 2-7

4-1 Calculated Values of the Inverse Relaxation Length for Americium-241 . . . . 4-12

4-2 Calculated Values of the Inverse Relaxation Length for Cobalt-60 . . . . . . . . 4-13

4-3 Calculated Values of the Inverse Relaxation Length for Cesium-137 . . . . . 4 4-14

4-4 Ratios of Plutonium-239, -240 to Americium-241 in Radionuclide Inventory and Distribution Program Soil Sampies .................. . . 4-15

4-5 Ratios of Strontium-90 to Cesium-137 in Radionuclide Inventory and Distribution Program Soil Samples

5-1 Comparison of Aerial and Radiochemical Plutonium Sampling Locations, Double Tracks, Nellis Air Force Range . . . . . . . . . . . . . . . . 5-5

5-2 Comparison of Aerial and Radiochemical Plutonium Sampling Locations Using an Alternate Summing Approach, Double Tracks, Nellis Air Force Range . . . . 5-6 


\section{List of Tables}

Number

Title

Page

3-1 Sites Studied by the Nevada Applied Ecology Group . . . . . . . . . . . . . . 3-2

3-2 Grid Size for Field Instrument for the Detection of Low-Energy

Radiation Readings at Nevada Applied Ecology Group Study Sites

3-3 Summary of Soil Samples from Nevada Applied Ecology Group Intensive Study Sites

4-1 Summary of Upper Limit Values from Radionuclide Inventory and Distribution Program Measurements 4-6

4-2 Sample Sizes of Radionuclide Inventory and Distribution Programs Surveys . . . 4-19 


\section{List of Acronyms and Abbreviations}

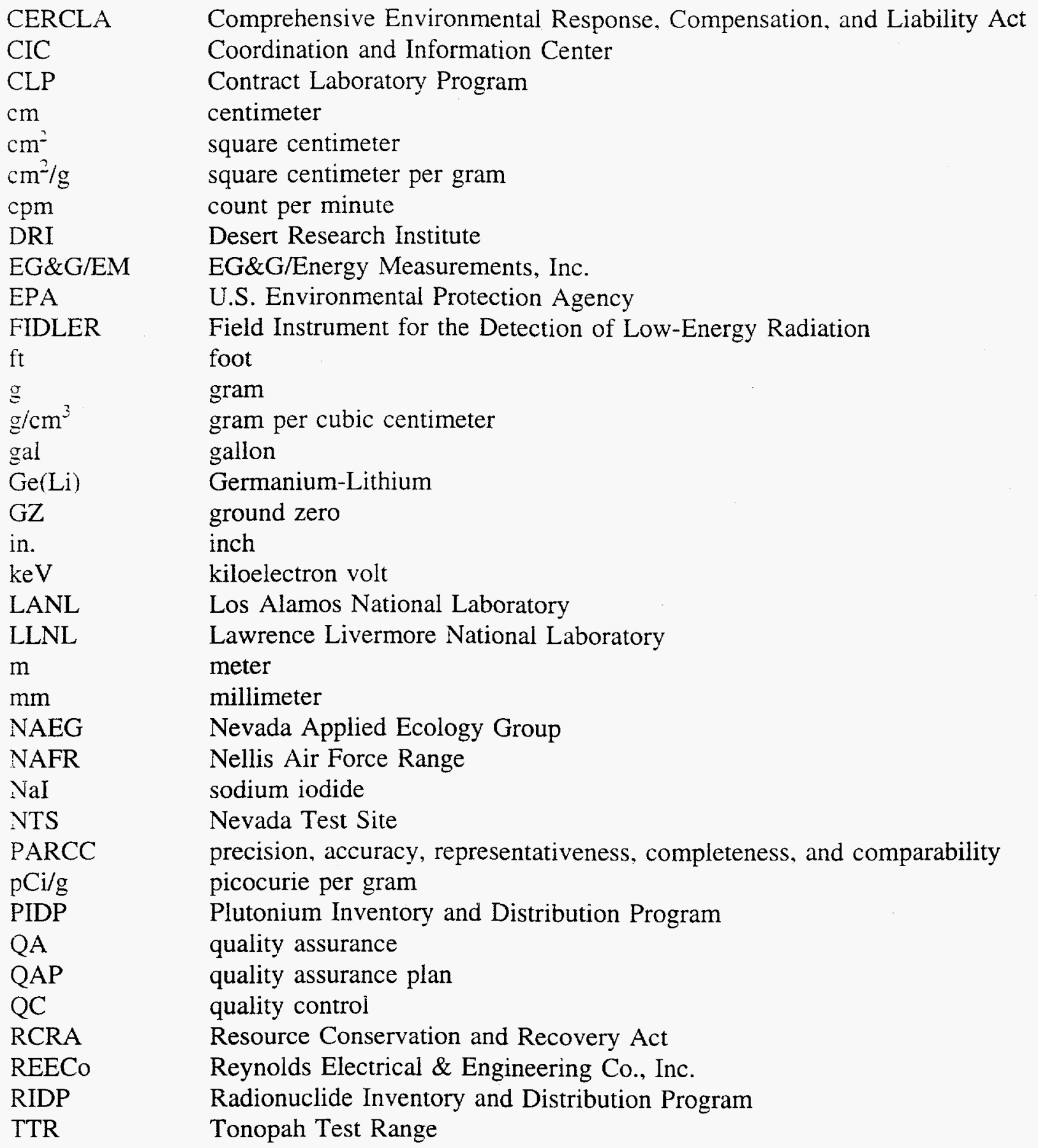




\subsection{Introduction}

Since 1951, 933 nuclear tests have been conducted at the Nevada Test Site (NTS) and test areas on the adjacent Tonopah Test Range (TTR) and Nellis Air Force Range (NAFR). Until the early 1960s, the majority of tests were atmospheric, involving detonation of nuclear explosive devices on the ground or on a tower, suspended from a balloon or dropped from an airplane. Since the signing of the Limited Test Ban Treaty in 1963, most tests have been conducted underground, although several shallow subsurface tests took place between 1962 and 1968. As a result of the aboveground and near-surface nuclear explosions, as well as ventings of underground tests, destruction of nuclear devices with conventional explosives, and nuclear-rocket engine tests, the surface soil on portions of the NTS has been contaminated with radionuclides. Figure 1-1 outlines the location of nuclear tests that may have potentially contaminated NTS soil.

Relatively little consideration was given to the environmental effects of nuclear testing during the first two decades of operations at the NTS. Since the early 1970 s, however, increasingly strict environmental regulations have forced greater attention to be given to contamination problems at the site and how to remediate them. One key element in the current environmental restoration program at the NTS is determining the amount and extent of radioactivity in the surface soil.

The general distribution of soil radioactivity on the NTS is already well known as a result of several programs carried out in the 1970s and 1980s. However, questions have been raised as to whether the data from those earlier studies are suitable for use in the current environmental assessments and risk analyses. The questions primarily focus on two issues: are the data sufficiently precise to provide estimates of radionuclide distributions, and are the data considered reliable for risk assessment purposes based on current quality assurance standards for sampling and analysis under the Resource Conservation and Recovery Act (RCRA) and the Comprehensive Environmental Response, Compensation, and Liability Act (CERCLA)?

The primary purpose of this preliminary data review is to determine to what extent the historical data collected at the NTS can be used in the characterization/remediation process. The following is a review the historical soil data from the NTS with respect to sampling locations, sampling and analysis methods, and current quality assurance protocols. As the 


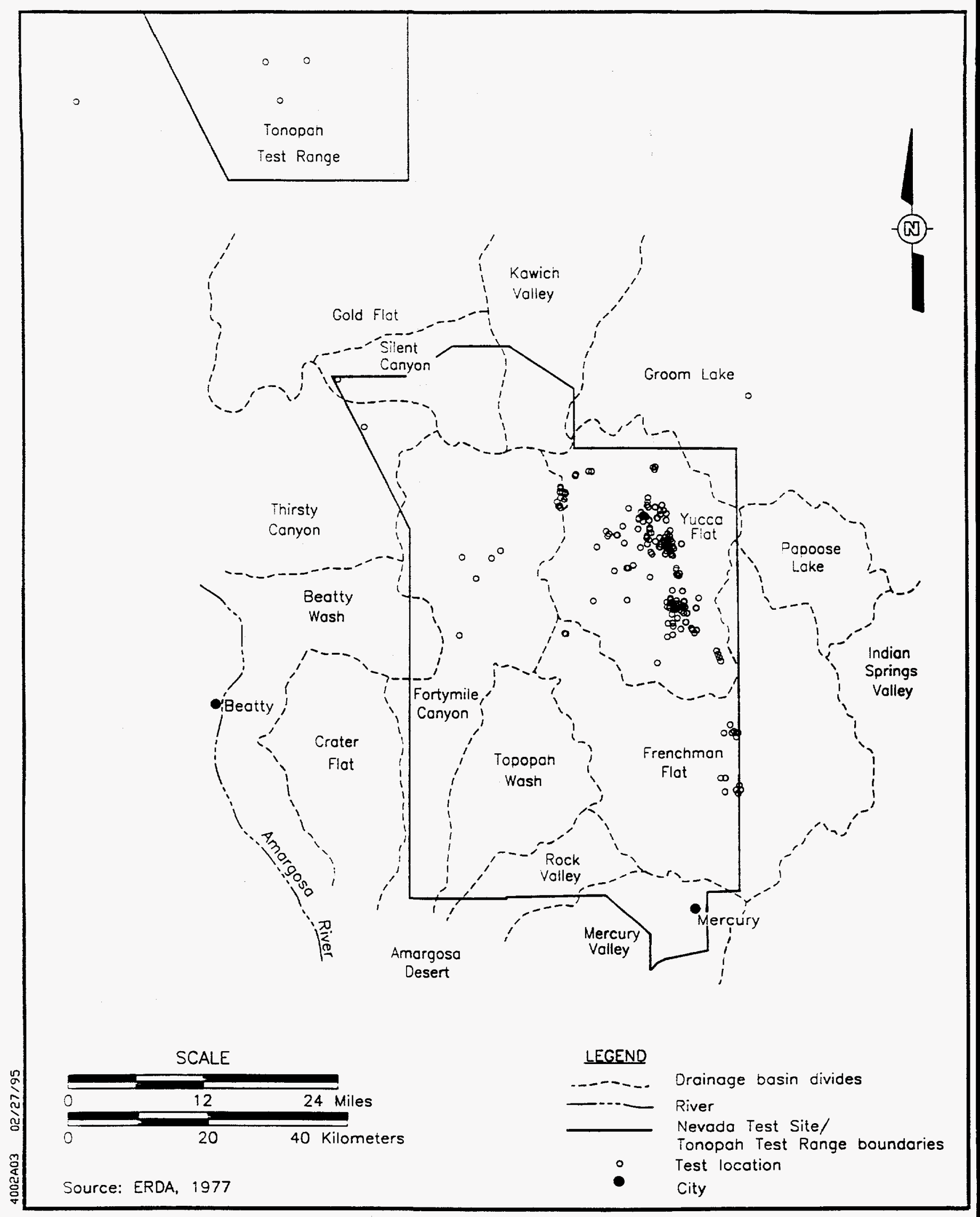

Figure 1-1

Potential Plutonium-Contaminated Soil Sites at Nuclear Test Locations 
historical data probably consist of more than 15,000 measurements of soil radioactivity, the results of this review are of considerable importance in a preliminary determination of data usability for site characterization, risk analysis, and remediation efforts. As the Soils Media Project progresses further into individual site characterization and remediation, the results of this effort will assist in deciding the number and type of additional samples that may be required to satisfy the needs of the data users.

Chapter 2.0 of this report contains an overview of the major soil radiological characterization programs that have occurred on the NTS, TTR, and NAFR. Chapter 3.0 focuses on the activities carried out as part of the Nevada Applied Ecology Group (NAEG) during the 1970s. The programs and procedures undertaken during the 1980s under the auspices of the Radionuclide Inventory and Distribution Program (RIDP) are described in Chapter 4.0. A discussion of data useability is contained in Chapter 5.0, and references may be found in Chapter 6.0. 


\subsection{Historical Data Overview}

The historical data are comprised of data collected since the early-1970s by various DOE contractors. Because the end uses of the data may have differed, analytical techniques used over the course of the investigations varied. The following section briefly describes the types of programs that were undertaken at the NTS.

\subsection{Aerial Surveys}

Between 1975 and 1984. EG\&G Energy Measurements. Inc. (EG\&G/EM), made aerial radiological surveys of a large portion of the NTS and surrounding areas (Figure 2-1). The aerial measurement system used an array of sodium iodide ( $\mathrm{NaI}$ ) detectors mounted on a helicopter. The helicopter flight lines were spaced 46 to 100 meters $(\mathrm{m})$ apart and were flown at altitudes of 30 to $60 \mathrm{~m}$ above ground level, depending on the area being surveyed. Previous aerial surveys of the NTS had been made with fixed-wing aircraft; the greater maneuverability and slower speed of the helicopter allowed a larger area to be surveyed and improved the sensitivity and spatial resolution of the measurements.

During the surveys, the position of the helicopter and the radiation data were recorded simultaneously. The data were used to produce maps showing isopleths of exposure rate due to radionuclides in the soil. In many areas, the concentrations of man-made radionuclides, such as cobalt-60 $\left({ }^{60} \mathrm{Co}\right)$, cesium-137 $\left({ }^{137} \mathrm{Cs}\right)$, and americium-241 $\left({ }^{241} \mathrm{Am}\right)$ could be inferred from the radiation data and plotted as well.

Because of the wide field of view of the airborne system, the data represent averages over a large area. The intensity of highly localized sources of radiation is typically underestimated by the aerial survey system. Consequently, the aerial survey data, while extremely valuable for preliminary large-scale site identification and screening, do not appear to be useful for the more precise mapping of individual radionuclide distributions required for site characterization and risk assessment purposes.

\subsection{Nevada Test Site Radiological Assessment Project}

In 1976, in situ spectrometry was adopted as the primary measurement method for large-area radiological surveys at the NTS. A vehicle-based measurement system was developed during 1977, and a detailed survey (100- and 200-feet [ft] grids) of Frenchman Flat in Area 5 was 


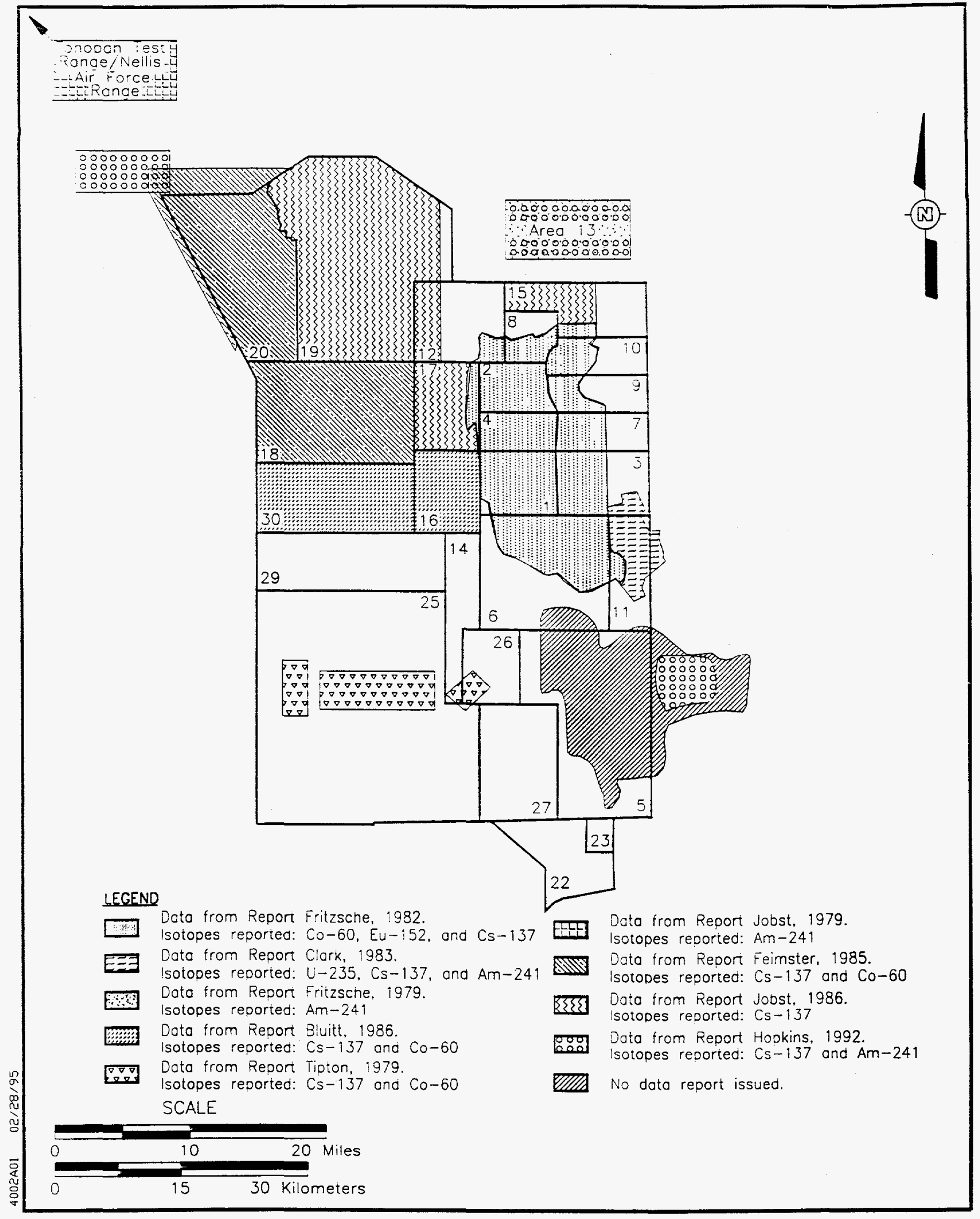

Figure 2-1

Nevada Test Site EG\&G Aerial Survey Summary 
completed in 1978 and 1979. Nine hundred and seventy-six spectra were recorded and analyzed for ${ }^{2+1} \mathrm{Am},{ }^{60} \mathrm{Co},{ }^{137} \mathrm{Cs}$, europium-152 ( $\left.{ }^{152} \mathrm{Eu}\right)$, and europium- 155 ( $\left.{ }^{i 55} \mathrm{Eu}\right)$; data for plutonium-239 $\left({ }^{239} \mathrm{Pu}\right)$ were obtained using $\mathrm{Pu} / \mathrm{Am}$ values from soil samples. The results were reported by Barnes et al. (1980), but are open to question because the detectors apparently were improperly calibrated (Anspaugh and Kordas, 1980).

Additional spectra were recorded in Area 5, Yucca Flat, and along the NTS boundary until the project was suspended in February 1980. There is no indication that these spectra were ever analyzed.

The spectra from this project are still available and could be reanalyzed; however, questions regarding survey accuracy because of improper calibration still remain. In addition, most of the areas covered were later resurveyed with a more sophisticated system as part of the RIDP (see Section 2.4). The exception is seven transects east of Frenchman Flat on the NAFR, measured at $1.000-\mathrm{ft}$ and 1-mile intervals to track the contamination plume from the Smallboy test. These are the only known ground-based radioactivity measurements from this area. Because of the uncertainty related to accuracy, the Radiological Assessment Program data will not be considered further in this evaluation.

\subsection{Nevada Applied Ecology Group}

The NAEG was created in 1970 to coordinate the environmental programs of the office of effects evaluation and to comply with requirements of the National Environmental Policy Act of 1969. Its objective was to integrate ecological research at the NTS. Plutonium became the first concern, and activities of the NAEG included delineating the locations of plutonium contamination, determining the concentrations and rates of movement of plutonium in various ecosystem components, evaluating potential radiological dose from plutonium, and identifying areas and techniques for cleanup or treatment. The scope of the program was later expanded to include transuranics and other radionuclides, as well.

The NAEG was organized into several research elements, each of which applied state-of-theart techniques to studying a particular aspect of the interaction of radionuclides with the environment. Among these elements were distribution and inventory, soils, vegetation, small mammals, large mammals, resuspension, statistics, and modeling. The two elements most relevant to the subject of this document are distribution and inventory and soils. The objective of the distribution and inventory element was to assess the overall distribution of radioactivity on and near the NTS with emphasis on plutonium. The soils element studied the 
amount, location. and chemical and physical status of plutonium in the soil at "intensively studied" sites. The locations of these studies are shown in Figure 2-2. The NAEG studies are discussed in more detail in Section 3.0 of this document.

\subsection{Radionuclide Inventory and Distribution Program}

Between 1981 and 1986, the RIDP carried out a comprehensive radiological survey of the surface soil on the NTS. The objective of the program was to estimate (within a factor of two) the distribution and the total inventory of important man-made radionuclides of NTS origin in the soil. The primary method of measurement was in situ spectrometry; soil samples were also collected and analyzed to provide information on radionuclide ratios and depth distributions. Figures 2-3 and 2-4 outline soil surveys performed by the RIDP.

The RIDP surveyed all the ground zeros (GZ) of aboveground nuclear tests, the GZ of underground tests where significant amounts of radioactivity reached the surface, all safetyshot sites on the NTS, the nuclear-rocket test faciiities in Area 25. and all other accessible areas on the NTS where aerial surveys showed elevated levels of radioactivity. Estimated inventories and distributions were reported for 14 manmade radionuclides. Section 4.0 of this document further details the RIDP activities. 


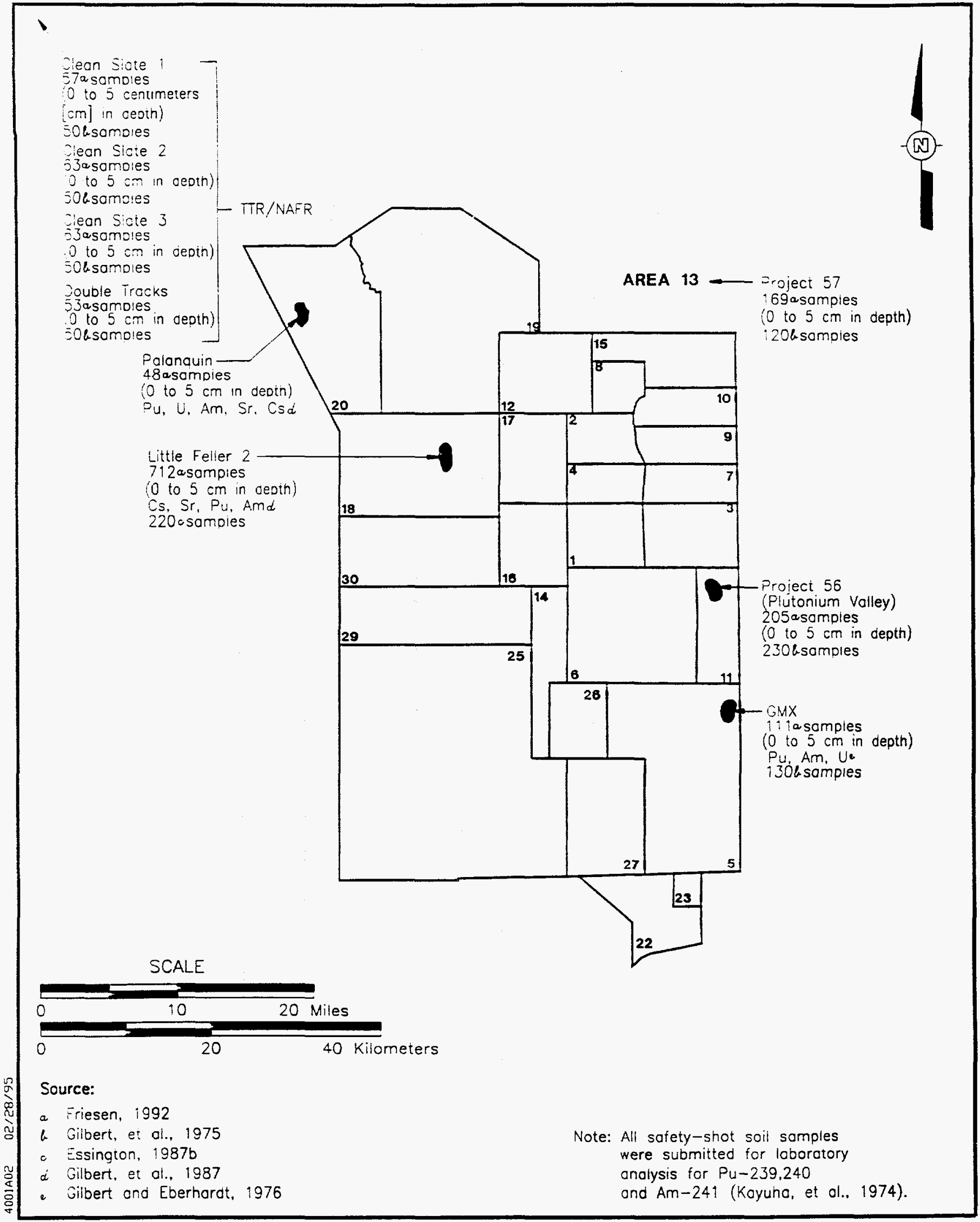

Figure 2-2

Nevada Test Site

Nevada Applied Ecology Group Soil Sample Summary 


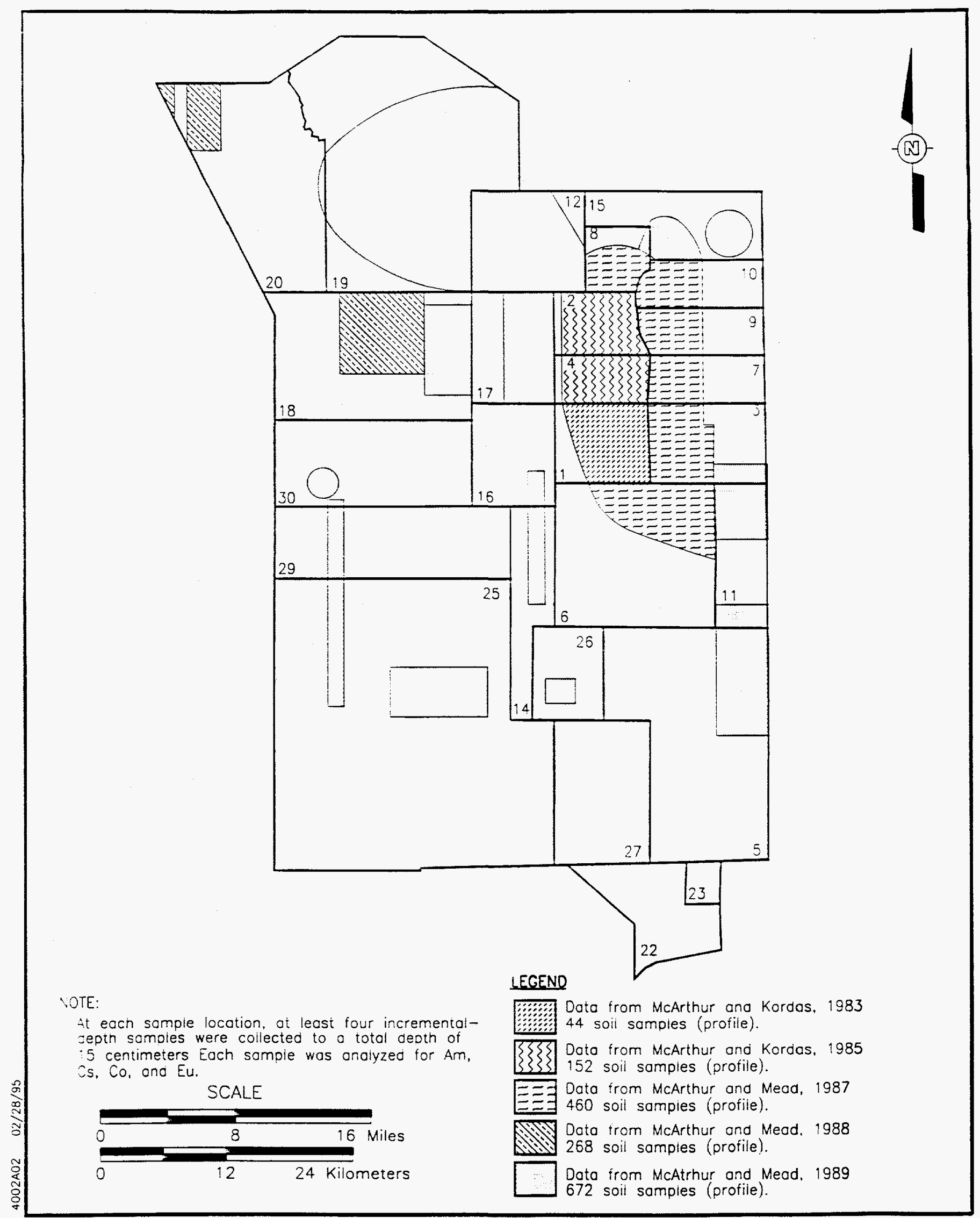

At each somple location, at least four incrementaleptn somoles were collected to o totol deoth of

5 centimeters Each somple was analyzed for Am, $s$, Co, and Eu.

$$
\text { SCALE }
$$




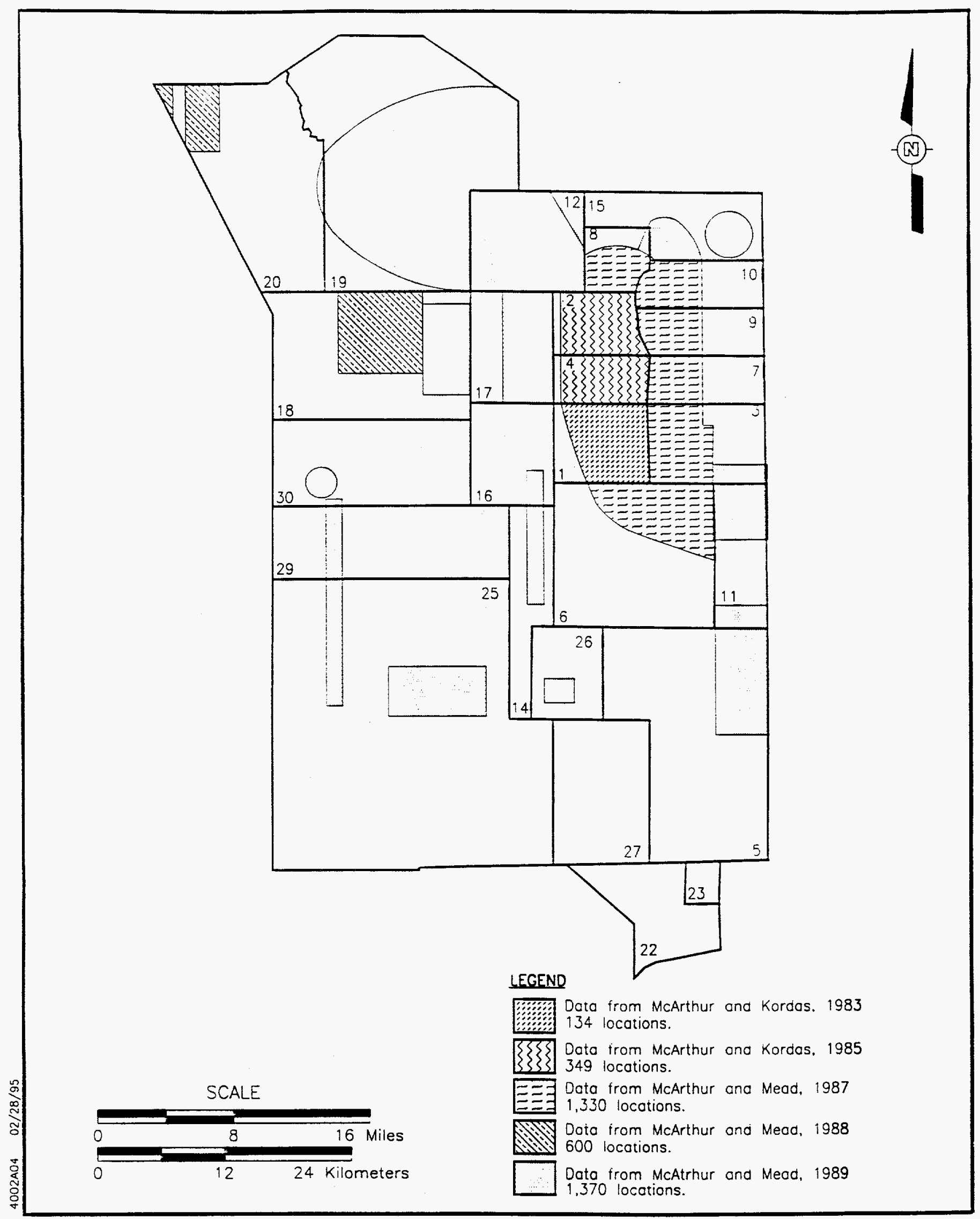

Figure 2-4

Nevada Test Site

Radionuclide Inventory and Distribution Program In Situ Measurement Location Summary 


\subsection{The Nevada Applied Ecology Group Program}

The NAEG was organized into several research elements devoted to studying the interaction of radionuclides with the environment. The two elements most significant to the present task of soil characterization are the distribution and inventory element and the soils element.

The distribution and inventory element of the NAEG (also referred to as the Plutonium Inventory and Distribution Program [PIDP]) began assessing plutonium levels on the NTS in 1973. The objective of this element was to assess the overall distribution of plutonium on and near the NTS. This element was executed through a series of phases: an historical review phase, an overview survey phase, and a comprehensive survey phase (Church et al., 1974).

The NAEG soils element objective was to study the amount. location, and chemical and physical status of plutonium in the soil at the study sites. The soils element was later expanded to include the study of other radionuclides at the sites of nuclear explosions. This element differed from the PIDP in that a few selected sites were studied rather than sites throughout the test area.

Because the original emphasis of the NAEG was on plutonium, its first studies were carried out at safety-shot sites, where conventional explosives had dispersed plutonium over a relatively small area. In the late 1970s, the program was expanded to include other long-lived radionuclides, and additional studies were done at sites where fission had occurred (nuclear sites). All the sites studied intensively by the NAEG are listed in Table 3-1, and Figure 2-2 shows those sites where soil samples were collected.

The NAEG intensive study sites first included only safety-shot sites because of the simplicity of their radiological and ecological characteristics. The first sites chosen for study were Projects 56 and 57. Project 56 in Area 11 was chosen because the site was best known radiologically, and the area could be maintained as an undisturbed site for studies of natural conditions. The site was thought to be an ideal area in which to study the resuspension, distribution, and translocation characteristics of plutonium under existing conditions and the lateral and downward movement of plutonium in soils by the action of surface waters (Essington, 1987a). 
Table 3-1

Sites Studied by the Nevada Applied Ecology Group

\begin{tabular}{||l|l||}
\hline \multicolumn{2}{|c||}{ Safety-Shot Sites } \\
\hline Area 5 & GMX \\
\hline Area 11 & Plutonium Valley, Sites A, B, C, and D (Project 56) \\
\hline Area 13 & Nellis Air Force Range (Project 57) \\
\hline Area 52 & Clean Slates 1, 2, and 3 Tonopah Test Range \\
\hline Area 71 & Double Tracks Nellis Air Force Range \\
\hline & \multicolumn{1}{|c|}{ Nuclear Sites } \\
\hline Area 2 & How, Badger, Turk, Whitney, Shasta. and Diablo \\
\hline Area 10 & Sedan \\
\hline Area 18 & Little Feller II (NS-201) \\
\hline Area 20 & Palanquin/Cabriolet (NS-219 and NS-221) \\
\hline
\end{tabular}

Project 57 in Area 13 was chosen to conduct inventory and distribution measurements and to evaluate plutonium assimilation by grazing animals in a contaminated area large enough to support livestock grazing. Evaluation of the radionuclide inventory and distribution at Area 13 and at TTR was considered important because these sites were not within the controlled boundary of the NTS and the feasibility of site cleanup was being considered. In addition, experiments were conducted to determine how man's activities influence resuspension and to explore methods and effectiveness of treatment and clean-up activities (Essington, 1987a).

The GMX site in Area 5 was later incorporated as one of the intensive study sites; the site had previously been chosen for resuspension studies and represented ecosystems different from those of Projects 56 and 57. There were 22 individual consecutive experiments conducted at the same GZ in Area 5. The plutonium used in the GMX experiments was not machined for use in typical nuclear devices as had been the case in Area 11 but were designed for physics "equation of state" experiments (Essington, 1987a).

By early 1972, interest grew in the Roller Coaster safety-shot sites in Area 52 (TTR). The range was being used for commercial grazing. Small areas of higher contamination were fenced; however, the grazing animals, as well as herds of wild animals, had access to contaminated materials. Characterization of the contaminant sources provided controlled 
characteristics and different ecosystem attributes compared to the other sites chosen for study. Thus, Clean Slates 1.2, and 3 and Double Tracks were included as intensive study sites for the NAEG (Essington, 1985).

By 1976, source-term information had been obtained relative to plutonium, americium, and uranium distributions at the safety-shot sites; efforts were then directed to the more complex nuclear sites. The presence of long-lived radionuclides contained in fused surface materiais, dispersed in various particulate configurations, and situated in a generally more rugged terrain (rocky soils) constituted a complexity not seen at the safety-shot sites. The nuclear sites designated by NAEG for intensive study were tower shot sites in Area 2 (How, Badger, Turk, Whitney, Shasta, and Diablo), Sedan site in Area 10, Little Feller II site in Area 18, and Palanquin/Cabriolet site in Area 20 (Essington, 1987a).

By the early 1980s, the NAEG had accomplished most of its major goals. Most field studies were stopped by 1983, and further efforts were largely devoted to synthesizing the results and publishing them in open literature. The program formally ended in September 1986.

\subsection{Data Collection}

\subsubsection{Distribution and Inventory Element}

The distribution and inventory element was executed through a series of phases. The first phase was a historical review to identify events and locate and review existing measurements of contamination at each event site. The second phase was an overview survey to determine general conditions at each site. The final phase was an intensive survey of each site based on information gained during the first two phases (Church et al., 1974).

During the overview survey, the area around each GZ was surveyed with portable GeigerMueller instruments to determine a "hot line," the path of highest radiation intensity resulting from fallout at the time of the original event. If the measurements did not conclusively establish a hot line, fallout maps were consulted to determine the probable direction of highest fallout. A transect was established along the hot line, and the exposure rate was measured every $100 \mathrm{ft}$ along the transect from GZ to $500 \mathrm{ft}$ and every 1,000 ft thereafter until exposure rates fell to less than 0.2 millirad per hour. These measurements were supplemented by alpha radiation measurements using an Eberline PAC-3G instrument (Church et al., 1974). 
Each hot-line transect measurement point was staked and marked for soil sample collection. At each stake, a hole was dug more than 30 centimeters $(\mathrm{cm})$ deep, and samples were collected by inserting a 10 - by 10 - by $2.5-\mathrm{cm}$ scoop horizontally into a vertical face of the hole. Three samples were collected from each hole: a surface sample from the surface to a depth of $2.5 \mathrm{~cm}$, a $15-\mathrm{cm}$ sample from 15 to $17.5 \mathrm{~cm}$, and a $30-\mathrm{cm}$ sample from 30 to $32.5 \mathrm{~cm}$. The collection scoop was decontaminated with alcohol after each use to prevent transfer of contaminants to succeeding samples. The scoop was designed to sample a constant area of 100 square centimeters $\left(\mathrm{cm}^{2}\right)$ and yield sample weights of approximately 500 grams $(\mathrm{g}$ ) although the weight varied widely due to differing soil composition and density (Church et al., 1974).

Through January 1974, 210 of 226 event sites were surveyed in the overview phase, resulting in collection of approximately 2,100 soil samples. The results of the overview phase are not published in any of the NAEG reports. The 1974 NAEG report (Church et al., 1974) refers to a separate comprehensive progress report "in preparation," but no such report has been found.

\subsubsection{Plutonium Inventory and Distribution Program Intensive Soil Sampling Phase}

The intensive sampling phase began in 1974 at Frenchman Flat in Area 5. Sample locations were selected both randomly and on a grid to determine which of these methods would provide a better estimate of distribution and inventory of radionuclides in areas where soil had been extensively disturbed by mechanical means (Brady and Church, 1975).

The grid sampling used a minimum grid distance of $100 \mathrm{ft}$, starting from the surface GZ out until radioactivity, based upon portable instrument measurements and previously obtained soil samples, declined to less than 0.1 nanocurie per gram. The grid distance was increased to $200 \mathrm{ft}$ in areas of less contamination. The total size and shape of the sampling grid was based primarily on the overview transect samples (Brady and Church, 1975).

The one randomly sampled location (Hamilton) was gridded in 200-ft squares measured from the surface GZ. Sample locations were measured from the southwest corner of each $200-\mathrm{ft}$ square using a table of random numbers to select distances east and north of the corner stake. Two such randomly selected locations were obtained from each grid square (Brady and Church, 1975). 
Once the sampling locations had been identified. metal stakes were driven into the ground, and metal tags were attached to the stakes to identify each location. The actual samples were obtained at a distance of 30 inches (in.) and an azimuth of $30^{\circ}$ from each stake to avoid taking the surface samples in ground disturbed during surveying and staking (Brady and Church, 1975).

After a surface sample had been collected, a backhoe was used to dig a trench 24 in. wide, $4 \mathrm{ft}$ deep, and approximately $6 \mathrm{ft}$ long. A 10-cm-wide, 10-cm-long and 5-cm-deep sampling tool was then used to obtain samples from a wall of the trench at depths of 30,60 , 90 , and $120 \mathrm{~cm}$. The sampling tool was forced into the wall using an electrically driven pneumatic ram specially adapted for this operation. The need for such deep sampling was based on previous surveys and the fact that Area 5 had been extensively disturbed by excavation and backfilling over the years. Much of this area is also under water during several months of the year, adding to the uncertainty of depth distribution (Brady and Church, 1975). The results of the intensive sampling phase are not published in any of the NAEG reports.

\subsubsection{Soils Element}

The area to be surveyed at each intensive study site in the soils element was determined by historical reports and surveys conducted after test events. A boundary was established around the study site, usually extending along the fallout pattern. A grid system was set up within the boundaries. The grid size varied from site to site, as shown in Table 3-2, especially in areas of high radioactivity near GZ locations, in order to best determine stratum size and location. An exception to the grid system was at the GMX site in Area 5, where instrument readings were collected along 24 radii extending out from GZ at $15^{\circ}$ intervals (Gilbert et al., 1975)

At each grid node of the safety shot sites, a reading was made with a Field Instrument for the Detection of Low-Energy Radiation (FIDLER) adjusted to count the low-energy (59.5 kiloelectron volts [keV]) gamma ray emitted by ${ }^{241} \mathrm{Am}$. The amount of ${ }^{241} \mathrm{Am}$ present in the soil was an approximate indicator of the amount of ${ }^{239,240} \mathrm{Pu}$ present (when the ${ }^{239,240} \mathrm{Pu}$ to ${ }^{241} \mathrm{Am}$ ratio is applied) so that the FIDLER readings were an approximate measurement of the relative plutonium concentration in the immediate vicinity of the detector. The FIDLER readings (counts per minute [cpm]) were used to determine strata (or subregions) within the site boundaries. This was the first step in designing a stratified random sampling plan for estimating the plutonium inventory in soil. The goal was to define strata so that the variations in plutonium concentrations from soil sample to soil sample within each stratum 
Table 3-2

Grid Size for Field Instrument for the Detection of Low-Energy Radiation Readings at Nevada Applied Ecology Group Study Sites

\begin{tabular}{|l|c|c|}
\hline \multicolumn{1}{|c|}{ Site } & \multicolumn{1}{|c|}{$\begin{array}{c}\text { Low-Concentration } \\
\text { Grid Size (feet) }\end{array}$} & $\begin{array}{c}\text { High-Concentration } \\
\text { Grid Size (feet) }\end{array}$ \\
\hline \hline Area 11 & 400 & 50,25 \\
\hline Area 13 & 400 & 100 \\
\hline Clean Slate 1 & 200 & 25 \\
\hline Clean Slate 2 & 200 & 100,50 \\
\hline Clean Slate 3 & 200 & 25 \\
\hline Double Tracks & 200 & 25 \\
\hline NS-201* & 200 & 100 \\
\hline NS-219,221* & 1.600 & 800,400 \\
\hline
\end{tabular}

*Grids at nuclear sites were used to establish soil sampling locations.

would be smaller than the variations between strata. The strata were typically drawn at activity-level increments of $1,000 \mathrm{cpm} ; 2,000 \mathrm{cpm}$; and so forth. In this situation, stratified random sampling was likely to yield an estimate of inventory with greater precision than would result if a simple random sample were taken over the entire area to be inventoried (Gilbert et al., 1975).

After the strata were designated, a random collection scheme was established to collect soil samples in each stratum. Locations were chosen at random with either a table of random digits or a random number generator. A minimum of 10 samples were collected in each stratum (Gilbert et al., 1975).

Sampling locations at the nuclear sites were selected differently from those at the safety-shot sites. First, a survey was conducted with portable radiation survey instruments on a transect from the GZ along the main fallout pattern. After the initial survey, a grid pattern was established at each site to collect soil samples. Sample locations were not selected randomly as they were at the safety-shot sites. Samples were collected in the corners and the center of each grid (Rosenberry, 1994). The purpose of grid sampling was to collect enough samples to estimate the spatial pattern and inventory of radionuclides (Gilbert et al., 1977). 


\subsection{Sampling Techniques and Field Analytical Methods for Plutonium Inventory and Distribution Program and Soils Elements}

Initially, soil sampling procedures were developed and tested in a microplot study near Project $57 \mathrm{GZ}$ in Area 13. Samples from that plot served to evaluate sample collection techniques and sample preparation techniques, which resulted in the adoption of the trench and ring methods of sampling, and ball-milling and sieving sample preparations to reduce soil samples to a more uniform texture (Essington, 1985). Simultaneously, those tests were used to determine the need for vertical profiles and to give an estimate of the maximum depth necessary to sample. Samples generated from the tests were used to evaluate a series of analytical procedures to estimate plutonium in an economical, yet accurate and precise manner (Essington, 1978).

\subsubsection{Surface Sampling}

Surface soil samples were collected with a ring sampier (Rosenberry, 1994). The ring sampler was made of metal with a diameter of $12.7 \mathrm{~cm}$ and a depth of $5 \mathrm{~cm}$. It had a lip on the outer circumference so that the sample technician could apply foot pressure on the top of the ring to insert it into the soil only $5 \mathrm{~cm}$; the lip would be flush with the ground surface. The soil was removed with a disposable spoon and put into a plastic bag. The bag was tagged with sample information and then enclosed in another plastic bag. Surface soil sample weight collected with the scoop or ring was on the order of 200 to $800 \mathrm{~g}$ and varied with the type of soil sampled (Wireman and Kayuha, 1976).

\subsubsection{Profile Sampling}

Profile sampling was conducted within a trench. dug either by shovel or backhoe. Individual samples were removed in incremental layers. Profile samples collected within a trench were removed with a 10 - by 10 - by 2.5 -cm scoop. At the desired sampling location, a trench approximately $0.5 \mathrm{~m}$ wide by $1 \mathrm{~m}$ long was excavated to the desired total depth (usually $25 \mathrm{~cm}$ ). One face of the trench was at the actual sampling reference point. The sampling area was wetted to prevent fine soil particles from being suspended by local wind gusts (Essington, 1978). The sampling scoop was inverted and placed down into the side of the trench to collect the first 2.5-cm-depth increment. Samples were removed and placed in a plastic bag and tagged. A clean sampler was used for each profile sample collected to prevent cross contamination (Patton, 1994). The desired number of profile layers was 10 per trench; however, fewer may have been collected based on soil type, and in some cases, more than 10 were collected if a request for deeper sampling was made. 


\subsubsection{Decontamination}

Decontamination of sampling equipment was performed after all available clean samplers were used. Samplers would be cleaned with a solution of water and alcohol and wiped clean with a paper towel. A swipe sample would be collected from the sampler. The swipe would be counted for radioactivity in a portable swipe counter. The swipe results would indicate whether decontamination of the sampler was adequate. Additional decontamination would be performed if the swipe results indicated removable contamination (Rosenberry, 1994).

\subsubsection{Number of Samples}

The number of samples reported from each intensive study site is given in Table 3-3. These numbers have been extracted from the various NAEG reports, and they are not entirely consistent. Inconsistency may be due to the collection of samples for various studies that may not have been used to calculate radionuclide distributions and inventories, such as with microplot and mound samples.

\subsubsection{Instrumentation and Source Checks}

The FIDLER was coupled with a ratemeter, Model PRN-5, manufactured by Bicron. The PRN-5 had adjustments to collect measurements within five different gamma-energy channels. The two used were the $60 \mathrm{keV}$ and $122 \mathrm{keV}$. The gamma-radiation energy characteristic of ${ }^{241} \mathrm{Am}$ is $60 \mathrm{keV}$. The gamma energy characteristic of ${ }^{57} \mathrm{Co}$ is $122 \mathrm{keV}$ and is representative of world-wide-fallout gamma radiation energy. Source checks were performed on the FIDLER twice each day with two traceable sources purchased from Eberline. $\mathrm{A}^{57} \mathrm{Co}$ source was used to check the FIDLER's response to $122-\mathrm{keV}$ gamma radiation, and a ${ }^{241} \mathrm{Am}$ source was used to check response to $60-\mathrm{keV}$ gamma radiation. The instruments were checked in the morning before measurements were made and after lunch. The FIDLER had considerable drift due to the high temperatures that occur at the NTS (Rosenberry, 1994).

\subsubsection{Sample Preparation \\ PIDP Samples}

Soil samples collected as part of the PIDP were received in the soil laboratory, Building 790 , in Mercury, NTS, double-wrapped in plastic and individually labeled. Transmittal forms were completed in the field, listing information concerning approximate alpha, beta, and gamma activities at the surface of the sample. When received at the soil laboratory, the samples were segregated according to activity levels. The field radiation levels were double-checked, using a stabilized assay meter (SAM). All samples were processed within filtered hoods. Each 
Table 3-3

\section{Summary of Soil Samples from Nevada Applied Ecology Group Intensive Study Sites}

\begin{tabular}{|c|c|c|c|c|}
\hline \multirow{2}{*}{ Area } & \# Samples & \multicolumn{2}{c|}{$\begin{array}{c}\text { Number of Locations } \\
\text { Surface Profile }\end{array}$} & Reference $^{\text {a }}$ \\
\hline 13 & $400^{\mathrm{b}}$ & - & - & $142 / 8$ \\
\hline 13 & 293 & 173 & 12 & $153 / 360$ \\
\hline 5 & 265 & - & 13 & $142 / 8$ \\
\hline 5 & 247 & 117 & - & $153 / 360$ \\
\hline 11 & $51^{\mathrm{c}}$ & - & 23 & $142 / 8$ \\
\hline 11 & 669 & 434 & - & $153 / 362$ \\
\hline $\begin{array}{c}\text { Tonopah Test } \\
\text { Range }\end{array}$ & $123^{\mathrm{c}}$ & - & 5 & $142 / 8$ \\
\hline Double Tracks & 135 & 85 & 5 & $153 / 361$ \\
\hline Clean Slate 1 & 140 & 90 & 5 & $153 / 361$ \\
\hline Clean Slate 2 & 145 & 95 & 5 & $153 / 361$ \\
\hline Clean Slate 3 & 150 & 100 & - & $153 / 361$ \\
\hline Nuclear Site 201 & 712 & - & - & $224 / 105$ \\
\hline Nuclear Sites & - & 70 & & $272 / 381$ \\
\hline 219,221 & & & & \\
\hline
\end{tabular}

a NVO report number/page number

${ }^{b}$ This number also includes microplot study samples.

${ }^{\mathrm{C}}$ These samples were from transect sample locations at the four intensive study sites.

sample was weighed, dried for at least 12 hours at $105^{\circ} \mathrm{F}$, reweighed, and then ball-milled for 5 hours. Upon request, selected samples were also passed through a 100 -mesh $(<0.15-\mathrm{mm})$ sieve. The samples were then aliquoted into the amounts requested and packaged. Samples to be analyzed by the Reynolds Electrical \& Engineering Co., Inc. (REECo), Environmental Sciences Laboratory were transmitted for analysis. All others were packaged and shipped in accordance with U.S. Atomic Energy Commission and U.S. Department of Transportation regulations to other participating laboratories. The remaining portion of each sample was labeled and archived, pending future requests for additional analyses (Church et al., 1974).

\section{Soils Element Samples}

Soil samples collected as part of the Soils Element program were double packed in plastic bags and sent to the soils preparation facility in Mercury, Building 790 . The standard 
procedures for soil preparation are detailed in Wireman et al., 1976. At Mercury, soil library numbers and a library data card were assigned to each sample to permanently identify it. The samples were surveyed with a SAM to separate them into approximate ${ }^{241} \mathrm{Am}$ activity levels prior to preparation. The soils were then carefully prepared for weighing and drying within a laboratory hood. The soil samples were dried for 24 hours at $105^{\circ} \mathrm{C}$. reweighed, and put into 1-gallon (gal) paint cans containing 10 one-in. molybdenum steel grinding balls and placed in a ball mill where they were rotated at $140 \mathrm{rpm}$ for 5 hours. At this point in the procedure, a percentage of the samples collected from an intensive study site were selected at random and prepared in the following manner:

Approximately 60 percent of the original number of samples are aliquoted for analysis from the ball-milled fraction. A soil fraction from approximately 35 percent of the original number of samples, after ball-milling, is passed through a 100-mesh sieve, and aliquots of the soil fraction passing the 100-mesh sieve are taken for analysis. For approximately 3 percent of the original number of samples, an aliquot of the soil fraction passing and an aliquot of the soil fraction not passing the 100-mesh sieve are taken for analysis, also the weight of the soil fraction passing the 100-mesh sieve and the weight of the soil fraction not passing the 100-mesh sieve are recorded for this 3-percent sample group. Approximately 2 percent of the original number of samples are prepared by the same procedure as the 3-percent group, except that only the aliquot of soil passing the 100-mesh sieve is taken for inter-intra laboratory comparison aliquots. An aliquot of each sample is analyzed with a $\mathrm{Ge}(\mathrm{Li})$ [Germinium-Lithium] system, for a short period, to determine the approximate ${ }^{24 I} \mathrm{Am}$ activity (Wireman and Kayuha, 1976).

\section{Nuclear Site Samples}

Sample preparation for soil from the nuclear sites was slightly different in that, after drying, the soil was sieved through a 10 -mesh $(<2$-millimeter $[\mathrm{mm}])$ screen. The coarse fraction was weighed and stored, while the entire fine fraction was analyzed by gamma spectroscopy.

\subsection{Analytical Methods}

\subsubsection{Plutonium Inventory and Distribution Program Samples}

The following information summarizes the REECo Environmental Sciences Laboratory analysis method for soil samples. The prepared sample was digested in a solution of hydrofluoric, nitric, and hydrochloric acids. Boric acid was used to remove the fluoride ions. The system was reduced with sodium bisulfite. The plutonium was then picked up by a carrier, precipitated as a hydroxide, and separated from the residue. The plutonium hydroxide was dissolved with nitric acid and oxidized. The plutonium was then isolated by anion 
exchange. The plutonium was electrodeposited on a stainless-steel disk and quantified by alpha-spectroscopy using a silicon barrier detector and counting for 333 minutes (min) (Church et al., 1974).

Gamma and americium results were obtained by counting the sample on $\mathrm{Ge}(\mathrm{Li})$ counters. Counting times for americium and gamma were 20 and $100 \mathrm{~min}$, respectively (Church et al., 1974).

\subsubsection{Soils Element Samples}

Radiological analysis of the soils included wet chemistry analysis of ${ }^{239.240} \mathrm{Pu}$ and gamma spectroscopy for ${ }^{241} \mathrm{Am}$ and other gamma emitters.

The REECo Laboratory used hydrofluoric acid and nitric acids to dissolve the soil into solution. The soil solution was extracted in an ion-exchange column and plated and counted for alpha activity (Lee, 1994). The same method is currently used by the REECo Laboratory.

\subsubsection{Sample Storage}

NAEG samples are stored in the soils library in Building 2106 in Area 26. The building is a secured building; permission and arrangements must be made prior to access. The samples are contained in 6-ounce $(\mathrm{oz}), 16-\mathrm{oz}$, or 32-oz Nalgene ${ }^{\mathrm{TM}}$ bottles or 1-gal or 25-gal cans. Each sample has a designated library number that correlates to its library card. The library card contains all pertinent information about the sample (Britton and Sorom, 1981).

\subsection{Records and Custody}

\subsubsection{Documentation}

Documentation of field sampling results was performed with several variations of forms. There was not a standard form developed, but the forms did contain basic information: date, names of field technicians, sample location in Nevada grid coordinates (or distance from GZ), instrument reading, and type of sample collected.

Other documentation known to be generated, but examples of which have yet to be found, is outined in the report, Security Plan for Processing NAEG Sample Data from NTS Nuclear Event Sites (REECo, 1977). This report specifies the administrative procedures that were to be implemented when NAEG samples and associated data from NTS nuclear event sites were collected and processed by REECo. Data and parameters generated during sample collection 
were transferred to various forms within the soils laboratory: Sample Collection Data, Form RE-8650; Sample Preparation Data, Form RE-8649; Shipping Data, Form RE-8651; and Request for Laboratory Services, Form RE-1585.

\subsubsection{Sample Custogy}

NAEG procedures for sample custody were considered adequate for the conditions that existed; however, those procedures are not consistent with the more rigorous requirements and quality assurance (QA) protocols in effect today. Sample custody was initiated when samples were first collected for NAEG. Sample custody used documentation requirements generated by REECo for the handling and shipping of radioactive samples and assumed a corporate custody attitude as opposed to sample custody by an individual.

NAEG sample custody began with the collection of samples from locations identified by an investigator. The field crew (REECo) collected the sample and affixed a field sample identifier (tag) to the sample and also to the Field Sampling Form, which contained all the pertinent sampling data, e.g., location, field instrument radiation readings, and comments. The samples and the field forms were then delivered to the soil laboratory (REECo), and the samples were prepared for analysis. The soil laboratory technician maintained custody of the samples and of the sample records. The technician also assigned a library number to each sample, and this number was the primary identifier of the sample. Samples or aliquots of samples were sent to the analytical laboratories for assay. If the REECo analytical laboratory received the samples, custody was assumed not to have been transferred. All samples were accompanied by a Laboratory Request Form that noted the sample identification, other pertinent information, and the requested analyses (Essington. 1994).

Samples that were sent to independent laboratories were delivered as a batch (a few to perhaps a hundred samples per shipment). Shipping papers documenting the transfer of samples to the receiving laboratory were exchanged. A letter was sent to the receiving laboratory listing the samples and requested analyses. Generally, only aliquots of the library samples were released to the laboratories; those aliquots were disposed by the contributing laboratories upon completion of the analyses and acceptance of the results by the investigator. No further documentation was generated except for reports of analytical results (Essington, 1994). 


\subsection{Quality Assurance Program}

The majority of the discussion on the NAEG quality assurance program (QAP) was derived from a draft report written by Dr. E. H. Essington entitled Philosophy and Design of a Quality Assurance Program for the Nevada Applied Ecology Group (1994). This document describes the elements of the NAEG QAP, such as philosophy and procedures, and presents examples of tests conducted in support of the QAP.

The primary QA effort was the analysis of samples by independent laboratories with simultaneous analysis of replicate aliquots of a percentage of the samples by the referee laboratory, Los Alamos National Laboratory (LANL). This effort was supported by occasional sharing of sources and common matrix materials dosed with the appropriate radionuclides. Results of these tests were evaluated by the Referee for their acceptability and reliability and were then presented for interpretation of their applicability to the project investigator. The Referee's task was to conduct the above tests relative to testing sample collection, preparation, and analysis procedures and to evaluate results consistent with the needs of the specific NAEG investigations (Essington, 1994).

The Referee tracked analytical procedure performance based on comparison of the reported analytical results with those of other laboratories. Consistently low yields, high replicate variability, or consistent biases in results signaled the need to investigate a laboratory's analytical and data reporting procedures (Essington, 1994). Several laboratories were used to analyze soil samples in the referee program. Each laboratory had its own procedures for analyzing soils for the NAEG and its own method of computing counting errors associated with its determinations. In addition, the definition and computation of detection limits were not consistent in all laboratories, and there were differences in the way results were reported (Gilbert et al., 1974). Ideally, all laboratories should have used the same analytical and reporting procedures to ensure comparability of results.

Laboratory procedures for the radiological parameters were not consistent between participating labs in the referee program. Soils were analyzed at LFE Environmental Analysis Laboratory, Los Alamos Scientific Laboratory, REECo Environmental Sciences Laboratory, Eberline Instrument Corporation, and McClellan Central Laboratory (Bradey et al., 1978).

Evaluation of the results utilized whatever information was available relative to the sample, e.g., performance of reference blind materials, reproducibility of replicate results, constancy and validity of ratios of various radionuclides in the same sample, and parity with expected 
leveis of radionuclides based on the sampling location. It was considered the ultimate responsibility of the NAEG Principal Investigator to accept or reject data based on the tests and recommendations provided by the Referee (Essington, 1994).

\subsection{Availability of Data}

Data generated from the analysis of samples is retained in a data base at the Desert Research Institute (DRI). The sample numbers that have records in this NAEG data base include 6,319 soil; 2,132 vegetation; and 689 small animal. The data base contains 66,000 result records. It does not include large animal data or FIDLER measurements. Other sources of data include NAEG reports or information from the individual project investigators.

Laboratory analytical data were sent directly to the project investigators from the laboratory, until REECo requested that this practice discontinue. and then data were disseminated directly through REECo to the individual project investigators.

The 1975 PIDP progress report (Markwell and Church, 1977) notes that over 6,000 soil samples from 1,450 locations in Areas 1, 4, and 5 had been analyzed, but the results were not reported. All samples were $\mathrm{Ge}(\mathrm{Li})$ scanned for gamma-emitting radionuclides, but only about 5 percent $\left(\sim 300\right.$ ) contained detectable amounts of ${ }^{241} \mathrm{Am}(>1$ picocuries per gram [pCi/g]). Ten percent of the samples $(\sim 600)$ were analyzed for plutonium by wet chemistry, and all were above the detection limit of $0.01 \mathrm{pCi} / \mathrm{g}$ of $\mathrm{Pu}-239$. The $\mathrm{Ge}(\mathrm{Li})$ scans were used to infer the concentration of plutonium in the sample; it was reported by Markwell and Church (1977) to be several orders of magnitude less sensitive than the wet chemistry analysis, and as a result, the $\mathrm{Ge}(\mathrm{Li})$ scan analysis for these samples could only be used to determine an upper limit of plutonium concentration. Also, the resulting data were not considered sufficient to estimate the distribution of plutonium at more than one or two of the sites, and it was felt that to estimate inventories at the other sites, most of the remaining soil samples would need to be analyzed for plutonium by wet chemistry or other more sensitive instrumentation in order to estimate both an inventory and distribution of plutonium (Markwell and Church, 1977).

The Markwell report stated that current efforts included "evaluation of the program's sampling and analysis procedures." There is no evidence that further work on the distribution and inventory element was performed at the NTS, because later progress reports deal only with the off-site regions. Results from the on-site program were not reported in detail because the data are questionable due to analytical problems that started at an unknown time (Kordas and Anspaugh, 1982). It is assumed that due to the high cost of plutonium analysis 
and the limited budget, additional soil samples were not reanalyzed for plutonium by wet chemistry analysis. The original scope of PIDP soil analysis used the Ge(Li) scans as an alternate method of sample analysis since plutonium could be inferred from known ratios of ${ }^{239.240} \mathrm{Pu}$ to ${ }^{241} \mathrm{Am}$. There was a wide variability of the ratio, and there may have been additional problems determining a site-specific ratio (Brady and Church, 1975). Since the original anaiytical results of ${ }^{241} \mathrm{Am}$ were detectable in only 5 percent of the total samples, no reports were written that included the gamma analysis data.

PIDP data does not exist in the DRI NAEG data base. Records at the REECo Coordination Information Center (CIC) contain some original data printouts of the PIDP data. 


\subsection{Radionuclide Inventory and Distribution Program}

The primary objective of the RIDP was to inventory the major radionuclides of NTS origin in surface soil at the NTS. Inciuded within this objective were estimating both the total amount and the distribution of each radionuclide. The overall goal for precision was to have a final inventory estimate that was known with 95-percent confidence within, at most, a factor of two (Kordas and Anspaugh, 1982). The program was restricted to the NTS; it did not address contamination on the TTR and NAFR.

The general regions of the NTS where activities had contaminated the soil were known from historical records, aerial surveys, and ground-based radiological monitoring before the RIDP began. To complete the inventory within the allotted five years, the program's efforts were focused on these regions. Only a few confirmatory measurements were made in regions with background levels of soil radioactivity. (Background levels were defined as levels comparable to those resulting from global fallout, i.e., fallout from high-yield thermonuclear tests in the Pacific and tests conducted in other countries. The relatively low-yield fission tests conducted at the NTS contributed little to global fallout.) The total area surveyed was roughly a third of the total surface area of the NTS.

To help coordinate the logistics of such a large-scale project, field activities were carried out in 25 individual surveys. A survey typically covered a single GZ and the associated fallout plume, though in Yucca Flat the plumes from many tests overlap and the boundaries between surveys were arbitrary. The precise areas to be surveyed were determined from the most recent (1976 to 1984) aerial surveys. Once each area was defined, a set of measurement locations was chosen, and the radionuclide concentrations at each location were measured by in situ spectrometry. The results were analyzed along with supplementary data from soil samples to estimate the inventory and distribution of 16 manmade radionuclides $\left({ }^{60} \mathrm{Co},{ }^{90} \mathrm{Sr}\right.$, ${ }^{101} \mathrm{Rh},{ }^{102 \mathrm{~m}} \mathrm{Rh},{ }^{125} \mathrm{Sb},{ }^{133} \mathrm{Ba},{ }^{134} \mathrm{Cs},{ }^{137} \mathrm{Cs},{ }^{152} \mathrm{Eu},{ }^{154} \mathrm{Eu},{ }^{155} \mathrm{Eu},{ }^{174} \mathrm{Lu},{ }^{238} \mathrm{Pu},{ }^{239.240} \mathrm{Pu}$, and $\left.{ }^{241} \mathrm{Am}\right)$.

The methods and results of the RIDP were published in a series of five reports (McArthur and Kordas, 1983 and 1985; McArthur and Mead, 1987, 1988, and 1989). A final report (McArthur, 1991) updated and summarized the results of the program. 


\subsection{In Situ Radiological Surveys}

\subsubsection{Equipment}

The key element of the in situ measurement system was a high-purity germanium detector that measured the gamma rays emitted from radionuclides dispersed in the surface and near-surface soil. The detector and a collimator shield were attached to the end of a telescopic mast fixed to the back of a vehicle capable of off-road travel (either a Chevrolet Suburban or an Imp, a tracked vehicle). In and on the vehicle were the other components of the measurement system, including a 4.096-channel pulse-height analyzer, a Hewlett-Packard 9831A desktop computer, a generator to provide power, and the receiver/transmitter of a microwave ranging system.

Several detectors of different types (P-type planar, P-type coaxial. and N-type coaxial) were used at various times during the project. Each detector was capable of high resolution, typically $1 \mathrm{keV}$ to $2 \mathrm{keV}$ full width at half maximum of detected photopeaks. The high resolution increased the ability of the system to quantify photopeaks and identify the radionuclides present. Each detector was individually calibrated.

Measurements in areas of high concentration gradients, as indicated by aerial surveys, were made with a lead and cadmium collimator shield attached to the detector to limit its field of view. These areas were primarily located near GZs. The collimator restricted the detector view to a small circular area on the ground. The physical angle of the cone was $50^{\circ}$ from the vertical; however, the cut-off angle at which gamma rays could not enter the detector was approximately $60^{\circ}$ for gamma rays with energies between $0 \mathrm{keV}$ and $300 \mathrm{keV}$. Shielding was not perfect for gamma rays with energies greater than $300 \mathrm{keV}$. A fraction of the total flux (depending on the incident energy) measured by the detector was from angles greater than $60^{\circ}$.

Measurements were made with the telescopic mast extended so the detector was positioned $24 \mathrm{ft}(7.4 \mathrm{~m})$ above the ground. When the detector was fully raised, its collimated field of view for 90 -percent response had a radius of about $10 \mathrm{~m}$ at $60 \mathrm{keV}\left({ }^{241} \mathrm{Am}\right), 12 \mathrm{~m}$ at $662 \mathrm{keV}$ $\left({ }^{137} \mathrm{Cs}\right)$, and $21 \mathrm{~m}$ at $1,332 \mathrm{keV}\left({ }^{60} \mathrm{Co}\right)$.

\subsubsection{Procedure}

Once the vehicle had been positioned at a measurement location, the mast was extended to raise the detector. The operator entered the date, time, and other information into the 
computer and began the data acquisition. During the 15-min acquisition time, gamma rays reaching the detector generated pulses that were fed into the pulse-height analyzer, which sorted them to produce a gamma-energy spectrum. After the measurement was complete, the spectrum was stored in the computer. The mast was then retracted and the detector secured before the vehicle moved to another location.

After the spectrum had been stored, it was analyzed by a computer program that evaluated specific photopeaks to determine the concentrations of important radionuclides. The results were printed out immediately and inspected by the operator to verify that the system was working properly. The spectrum was also written to magnetic tape for further analysis.

\subsubsection{Determining Measurement Location}

For most measurements, the position of the vehicle was determined with a Motorola MiniRanger III microwave ranging system, using a receiver/transmitter mounted on the vehicle and two or more remote reference stations whose positions were accurately known. The reference stations were repeatedly interrogated by the receiver/transmitter, and the average round-trip travel time of the signal was used to determine the distance to each reference station. Two such distances were sufficient to determine the location of the vehicle.

After the set of measurement locations for a survey had been selected, the distances of the locations from the available reference stations were computed. Those distances were given to the field crews, who positioned the vehicle as close as possible to each requested location. Often craters, washes, or similar obstacles prevented the exact location from being reached, but precise positioning of the vehicle was not considered crucial. The actual distances to the reference stations were recorded as each measurement was being made, and the actual location was computed during later data processing.

The error associated with the microwave ranging system included both geometric and statistical uncertainties. The geometric uncertainty was related to the angle between the receiver/transmitter and the two reference stations. It was smallest when the angle was $45^{\circ}$ and greatest when the angle was $0^{\circ}$ or $180^{\circ}$. The statistical uncertainty was due primarily to variations in signal propagation time in the atmosphere and electronic circuitry of the receiver/transmitter and the two reference stations. The manufacturer quoted a typical range accuracy of $\pm 2 \mathrm{~m}$. The combination of uncertainties led to a typical accuracy of $\pm 10 \mathrm{~m}$ at distances up to $60 \mathrm{~km}$ from the reference stations. 
An equipment failure prevented use of the microwave ranging system during part of the first RIDP survey in Area 1, so many measurement locations there were determined by surveyors. Some of the later surveys covered mountainous and forested regions where off-road travel was impractical. Measurements in those regions were made mainly along roads and jeep trails, with the locations determined using mileposts and odometer readings.

\subsubsection{Spectral Processing and Analysis}

The magnetic tape cartridges containing the spectra were taken to EG\&G/EM's offices in Las Vegas, where the contents of each cartridge were verified against the operator's log sheets. The cartridges were then taken to the DRI where the spectra were transferred to 9-track magnetic tapes. A computer program read each spectrum, calculated the Nevada Grid Coordinates of the measurement location from the distances to the reference stations, and printed out the location and all operator-entered information (e.g., date, time, comments, and so on). The printouts were checked, and the spectra were corrected as necessary to relate results of aerial surveys to meaningful values at ground level. Finally, the spectra were written to a new tape, which was sent to Lawrence Livermore National Laboratory (LLNL) for analysis.

The spectral analysis was done by a modified version of the GAMANAL program of Gunnink and Niday (1971). GAMANAL is a sophisticated gamma-ray analysis program that performs background delineation, peak search, peak fitting, and radionuclide identification. It uses a Gaussian curve with an exponential tail for peak fitting and can separate overlapping peaks. Modifications to the program for in situ spectrometry included eliminating the detector efficiency and source geometry calculations and replacing branching intensities with detector conversion factors. The output of the GAMANAL analysis of the RIDP spectra included the concentrations and percent error of 15 natural and manmade radionuclides $\left({ }^{40} \mathrm{~K},{ }^{60} \mathrm{Co},{ }^{101} \mathrm{Rh}\right.$, ${ }^{102 \mathrm{~m}} \mathrm{Rh},{ }^{125} \mathrm{Sb},{ }^{133} \mathrm{Ba},{ }^{134} \mathrm{Cs},{ }^{137} \mathrm{Cs},{ }^{152} \mathrm{Eu},{ }^{154} \mathrm{Eu},{ }^{155} \mathrm{Eu},{ }^{174} \mathrm{Lu},{ }^{232} \mathrm{Th},{ }^{238} \mathrm{U}$, and ${ }^{241} \mathrm{Am}$ ) although not all of these were reported from every surveyed area.

\subsubsection{Conversion Factors}

The conversion factors used by GAMANAL to convert counts in a photopeak to radionuclide concentration were computed separately for each detector. A simplified description of the computation is given in McArthur and Kordas (1983), and more detailed descriptions can be found in Anspaugh (1976) and Beck et al. (1972). For present purposes, it suffices to note that the results are affected by assumed values of several parameters: 
- Soil density - a wet density value of 1.5 grams per cubic centimeter $\left(\mathrm{g} / \mathrm{cm}^{3}\right)$ was used for all analyses. Wet density was measured at 11 locations near the Galileo GZ in Area 1 during the first RIDP survey. The average value of $1.6 \pm 0.1 \mathrm{~g} / \mathrm{cm}^{3}$ was considered acceptably close to the assumed value.

- Soil moisture content - a value of 10 percent was used. Measured values at the 11 locations in Area 1 where the wet density was measured averaged $8 \pm 2$ percent.

- Air density - the assumed value of $0.001204 \mathrm{~g} / \mathrm{cm}^{3}$ is the density of dry air at $20^{\circ} \mathrm{C}\left(68^{\circ} \mathrm{F}\right)$ and $760 \mathrm{~mm}$ Mercury.

- Attenuation of gamma rays by soil and air - mass attenuation coefficients (in square centimeter per gram $\left[\mathrm{cm}^{2} / \mathrm{g}\right]$ ) for all energies of interest were obtained by interpolating from values in Beck et al. (1972). As a check, the attenuation by a soil sample from Area 1 was measured at three energies. The results agreed closely with those of Beck et al., 1972.

Details of the soil measurements in Area 1 can be found in McArthur and Kordas (1983). No further tests of the assumed values were carried out. Normal variation in these parameters would introduce an error of no more than a few percent in the calculated radionuclide concentrations.

The conversion factors also depend heavily on the distribution of the radionuclide with depth in the soil. As in previous applications of the in situ method on the NTS (for example, Kirby et al., 1977), the distributions of man-made radionuclides were assumed to be exponential:

$$
I(z)=I_{s} \exp \{-\alpha z\}
$$

where $I(z)$ is the activity per unit volume at depth $z(\mathrm{~cm})$ and $I_{s}$ is the activity per unit volume at the surface. The depth distribution is characterized by the parameter $\alpha$, the inverse (reciprocal) of the relaxation length.

Values of $\alpha$ for various radionuclides in each survey area were calculated using data obtained from soil samples. Further details are given in Section 4.3.

The assumption of a depth distribution is generally considered the most important source of error in applying the in situ method (Kirby et al., 1977). The effect is most serious at low energies. At $60 \mathrm{keV}$, a change in $\alpha$ from 0.1 to $1.0 \mathrm{~cm}^{-1}$ can cause a fourfold difference in 
the detector response; at $1,332 \mathrm{keV}$, the difference is about twofold. The error also increases at the lower range of $\alpha$ values (0.05 to $0.2 \mathrm{~cm}^{-1}$; see Figure 6 in McArthur and Kordas, 1983).

\subsubsection{Detection Limits}

If the GAMANAL program did not find the peaks associated with a particular radionuclide, it calculated an upper limit value from the integrated background in the peak region and the detector conversion factor. These values varied from one survey area to another and from one detector to another, so there is no single "detection limit" for a given radionuclide. A statistical summary of the upper limit values reported for the six most important gammaemitting radionuclides is given in Table 4-1. The distributions are highly skewed to the right, so the median gives a better representation of central value than the mean.

Table 4-1

Summary of Upper Limit Values from Radionuclide Inventory and Distribution Program Measurements

\begin{tabular}{||l|c|c|c|c|c|c||}
\hline & \multicolumn{6}{|c|}{ Nanocuries Per Square Meter } \\
\hline Radionuclide & Number & Mean & $\begin{array}{c}\text { Standard } \\
\text { Deviation }\end{array}$ & Median & Minimum & Maximum \\
\hline \hline${ }^{241} \mathrm{Am}$ & 2,475 & 59 & 47 & 40 & 7 & 640 \\
\hline${ }^{60} \mathrm{Co}$ & 2,419 & 16 & 13 & 13 & 1.5 & 99 \\
\hline${ }^{137} \mathrm{Cs}$ & 247 & 24 & 12 & 22 & 4 & 116 \\
\hline${ }^{152} \mathrm{Eu}$ & 2,806 & 61 & 52 & 48 & 6 & 530 \\
\hline${ }^{154} \mathrm{Eu}$ & 3,549 & 86 & 99 & 69 & 4 & 1,010 \\
\hline${ }^{155} \mathrm{Eu}$ & 3,530 & 56 & 53 & 38 & 6 & 760 \\
\hline
\end{tabular}

\subsubsection{Records and Custody}

The only written records associated with the data collection were the log sheets filled out by the operators during daily field activities. Included on the log sheet was the most important information associated with each spectrum: the date, run number, detector number, acquisition time, microwave ranges, location of the spectrum file on the tape cartridge, and comments. This information was also recorded in the spectrum file. The log sheets were checked against the information in the file by supervisors at EG\&G/EM and by the data 
processing technician at DRI. The original log sheets, as well as the tape cartridges on which the spectra were recorded, are in the custody of EG\&G/EM.

The results of the GAMANAL analysis of each spectrum were sent to DRI. Those results were added to the descriptive information for each spectrum on 9-track magnetic tape. The GAMANAL output is in the custody of LLNL. The magnetic tapes containing the updated spectra are stored at the DOE/NV computer center.

\subsubsection{Data Handling}

The analysis of the in situ measurement results was carried out at DRI. The first step was to rearrange the files of GAMANAL results received from LLNL into data files containing the coordinates of the measurement location and the calculated radionuclide concentrations. This manipulation was done by reading and writing computer files, so there was virtually no chance for data entry errors. The output data files were randomly checked to ensure that the numbers had been transferred correctly.

Once the data files had been prepared, the data were plotted on a map and inspected for any highly unusual values. Such values were reported to the spectral analyst at LLNL, who attempted (successfully, in most cases) to verify the result. Occasionally, remeasurements or additional measurements were requested. Few such problems arose, however, because most of the questionable spectra were identified by EG\&G/EM before the tape cartridges were sent to DRI.

Further analysis of the data to estimate radionuclide inventories and distributions is described in the RIDP reports cited above. The details are not relevant to the present task.

\subsubsection{Quality Control}

Probably the most important element of quality control in making the in situ measurements was detector calibration. Before a detector was used in the field, it underwent extensive calibration in a laboratory. These calibrations were of two types. First, the effective area was measured at various energies by placing different sources underneath the detector and calculating the efficiency per unit of incident flux. Second, the response of the detector to gamma rays striking it from different angles $\left(0^{\circ}\right.$ to $\left.90^{\circ}\right)$ was determined. The angular response measurements were repeated using a series of sources of different energies. The calibration of each detector was repeated periodically, and the conversion factors were adjusted as necessary. After a detector was calibrated, it was taken to a reference location in 
Yucca Flat (usually Galileo \#4) and used to record a spectrum. which was compared to previous spectra from the same location.

Another type of calibration was carried out three times a day (morning, noon, and afternoon) during field operations. A mixed source containing ${ }^{2+1} \mathrm{Am},{ }^{137} \mathrm{Cs}$, and ${ }^{60} \mathrm{Co}$ was placed $30 \mathrm{~cm}$ under the detector, and a spectrum was recorded for $2 \mathrm{~min}$. The results were used to verify the resolution, relative efficiency, and energy gain of the system. The gain was adjusted as necessary to maintain $0.375 \mathrm{keV}$ per channel. The calibration spectra were also used later in the GAMANAL analysis to obtain peak-shape parameters needed for fitting the photopeaks.

About 30 percent of the 8,550 RIDP gamma-ray spectra were recorded during laboratory calibration of the detectors. Another 23 percent were daily field calibrations. The calibration spectra were recorded on tape and sent to LLNL along with the spectra recorded in the field.

Additional procedures for quality control were in place during data collection. While the spectrum was being recorded, the field crew set up a pressurized ionization chamber $1 \mathrm{~m}$ above the ground and measured the external exposure rate to gamma radiation. After the measurement was complete and the computer program had calculated the radionuclide concentrations, it converted those concentrations to equivalent exposure rates. It then summed the individual rates to determine the total exposure rate. Both the calculated and the measured values were recorded on the tape cartridge and printed out along with the individual radionuclide concentrations for inspection by the system operator. Because of the different fields of view of the detector and the ion chamber, the two values were only roughly comparable, and the comparison was qualitative. Problems with the ion chambers led to exposure rates being measured at only about 60 percent of the in situ measurement locations.

In addition to being checked immediately after it was recorded in the field, each spectrum was checked a second time in Las Vegas before it was sent to DRI for further processing. This second check was made by a senior scientist who was thoroughly familiar with the measurement system.

\subsection{Soil Samples}

Soil samples were collected and analyzed for two purposes: to determine the distribution of radionuclides with depth in the soil and to measure the concentrations of radionuclides that either do not emit strong gamma rays or have very low probabilities of emitting gamma rays and thus are not easily quantified using the in situ system. 


\subsubsection{Sample Collection}

Soil samples were typically collected at locations of in situ measurements along two perpendicular transects through the GZ, if one was present. The procedure was similar to that used to collect the NAEG profile samples. First. a pit was dug $30 \mathrm{~cm}$ deep with one face at the desired sampling location. The front 2.5 to $5 \mathrm{~cm}$ was then shaved off the face to expose fresh soil. This soil was sampled twice with a $2.5-\mathrm{cm}$ scoop, then twice again with a $5-\mathrm{cm}$ scoop, resulting in a profile of 4 increments to a total sampling depth of $15 \mathrm{~cm}$. At the Sedan site, where it was suspected that radioactivity extended deeper into the soil, eight profiles were collected in six $5-\mathrm{cm}$ increments to a total depth of $30 \mathrm{~cm}$. The RIDP soil samples were collected by the same REECo personnel who collected NAEG soil samples in the later stages of that program. The procedures for documentation and sample custody used for the NAEG sampies (Section 3.4) were also used for the RIDP soil samples.

\subsubsection{Sample Analysis}

Prior to analysis, each sample was weighed, dried in an oven, reweighed, and then homogenized with a ball-mill and sieved through a 10-mesh (2-mm) screen. The fine fraction was then analyzed by gamma-ray spectrometry. Analysis of the coarse fraction from a few samples from different areas showed that on average about 99 percent of the radioactivity was contained in the fine fraction (McArthur and Kordas, 1983; Essington, 1989).

Gamma-ray spectrometry was done at the REECo Analytical Laboratory during the early stages of the program. Later, the REECo Laboratory recorded a gamma-ray spectrum from each sample and sent it to LLNL to be analyzed using the GAMANAL program. The results were used at LLNL to estimate inverse relaxation lengths as described below.

The fine fraction of the top ( 0 to $2.5 \mathrm{~cm}$ ) sample of a few profiles from each area was also analyzed chemically. Each such sample was first analyzed by gamma-ray spectrometry for ${ }^{241} \mathrm{Am}$ and ${ }^{137} \mathrm{Cs}$. An aliquot was then totally dissolved, and the strontium and plutonium were chemically separated from the solution and quantified using alpha and beta spectrometry. The exact protocol varied somewhat during the course of the project; important modifications included the following:

- The samples from the first survey (the Galileo site in Area 1) were chemically analyzed for ${ }^{89} \mathrm{Sr}$, ${ }^{90} \mathrm{Sr}$, and ${ }^{239,240} \mathrm{Pu}$. All analyses were done by REECo.

- Later samples were not analyzed for ${ }^{89} \mathrm{Sr}$, which was not found in the Galileo samples, but were analyzed for ${ }^{238} \mathrm{Pu}$. 
- The radiochemical analysis of all samples collected after June 1983 was done by TMA/Norcal instead of REECo.

The analytical results of the surface samples were used primarily to calculate radionuclide ratios $\left({ }^{90} \mathrm{Sr}\right.$ to ${ }^{137} \mathrm{Cs}$, ${ }^{238} \mathrm{Pu}$ to ${ }^{241} \mathrm{Am}$, and ${ }^{239.240} \mathrm{Pu}$ to ${ }^{241} \mathrm{Am}$ ) that were in turn used to calculate the concentrations of strontium and plutonium at all the other in situ measurement locations. The gamma-spectrometry results also served to check the results of the gamma analysis of the full set of profile samples.

Palanquin/Cabriolet and Little Feller II were two of the sites intensively studied by the NAEG. New soil samples were not collected when the RIDP surveyed these areas. Instead, the results from several NAEG soil profiles were used to estimate inverse relaxation lengths. The top-increment samples from the same profiles were retrieved from storage and reanalyzed to determine radionuclide ratios.

\subsection{Inverse Relaxation Lengths and Radionuclide Ratios}

The estimation of the inverse relaxation length $\alpha$ was described by one of the scientists involved as "more of an art than a science." The process had two steps: calculating a value from each set of soil profile data and determining a single representative value to be used in the GAMANAL analyses.

In general, the calculation was done by integrating the exponential equation, plugging in the concentrations measured in the four (usually) increments, and solving for $\alpha$. Complications arose from the presence of upper limit values in the data and from the occurrence of relatively high concentrations in the lowest increment (meaning that the sample was not deep enough). The methods of dealing with such problems were modified somewhat during the project. Details can be found in McArthur and Kordas (1983) and McArthur and Mead (1987 and 1988).

As a rule, 10 to 15 soil profiles were collected in each contaminated area. After all the $\alpha$ values for an area had been calculated, they were often observed to fall naturally into two groups. Values from locations within a few hundred yards of GZ were typically low, indicating that radioactive materials had penetrated deeper into the soil. Regions farther away from GZ had higher values indicative of radioactivity concentrated nearer the surface. Therefore, in most surveys, a GZ region was defined on the basis of the calculated values, 
and the spectra from locations in the GZ region were analyzed using different $\alpha$ values than were used for the other locations.

Several sets of calculated $\alpha$ values for the three most important radionuclides are plotted in Figures 4-1 through 4-3 to illustrate the amount of variability encountered. In almost all cases, the range of values is quite large, making the selection of a representative value highly subjective. The exact method by which representative values were chosen is not well documented in the RIDP reports. The discussion in McArthur and Mead (1988) states that the representative value was based on the geometric mean of the observed values. However, as shown in the plots, some of the "representative" values were actually outside the range of the observed values (e.g., Diablo, Whitney, and Buggy in Figure 4-1).

The observed ratios of ${ }^{239.240} \mathrm{Pu}$ to ${ }^{2+1} \mathrm{Am}$ and ${ }^{90} \mathrm{Sr}$ to ${ }^{137} \mathrm{Cs}$ are plotted in Figures 4-4 and 4-5. Although the variability is large in a few cases, the range of values suggests that the average can be determined within a factor of two with a fairly high degree of confidence.

\subsection{Quality Assurance}

The analysis of the soil samples was accompanied by a set of QA procedures to help assess the reliability of the resuits. These procedures were minimal at the start of the RIDP, but were expanded into a relatively complete and formal QA program by about the midway point. Details are presented as appendices in RIDP reports 2 through 5. A draft summary report on the RIDP QA program has recently been prepared by the QA referee (LANL), but it was not available for this evaluation. The following discussion addresses only the QA procedures specific to the RIDP. No attempt has been made to obtain information on the internal QA programs used at the analytical laboratories (REECo and TMA/Norcal) at the time the RIDP samples were analyzed.

No information is available on the QA procedures in place for the analysis of the first 44 RIDP soil samples from the Galileo site in Area 1. The analysis of the next group of 152 samples from Areas 2 and 4 included replicate aliquots of some samples and aliquots of an independently calibrated reference soil. In addition, duplicate gamma analyses of some samples were carried out at LLNL. (The numbers of such aliquots and duplicate analyses were not reported.) The results showed several inconsistencies (apparent high and low outliers and biases). To help resolve those inconsistencies, five samples were submitted to 


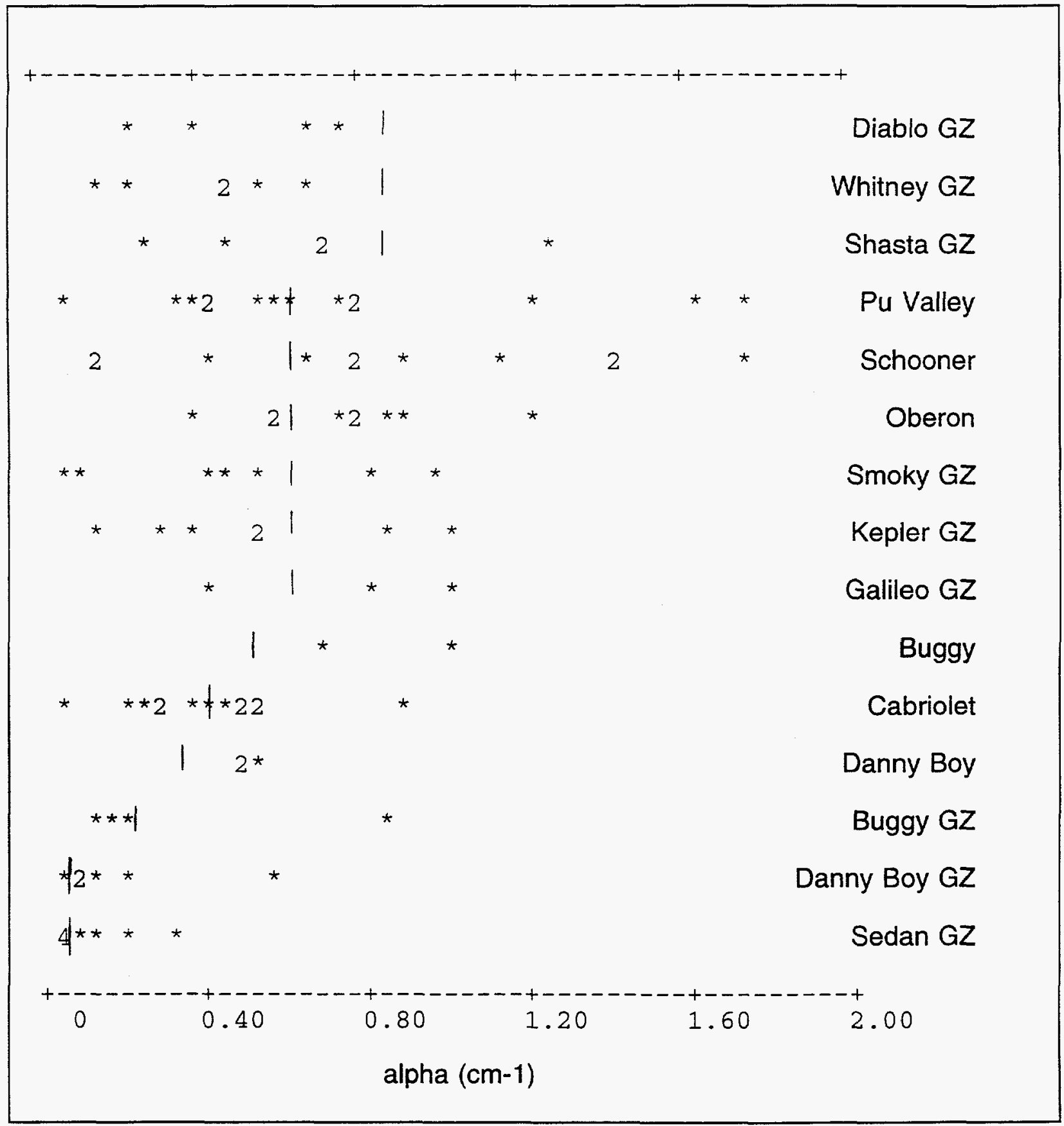

The vertical lines show the values used in analyzing the Radionuclide Inventory and Distribution Program spectra.

Figure 4-1

Calculated Values of the Inverse Relaxation Length for Americium-241 


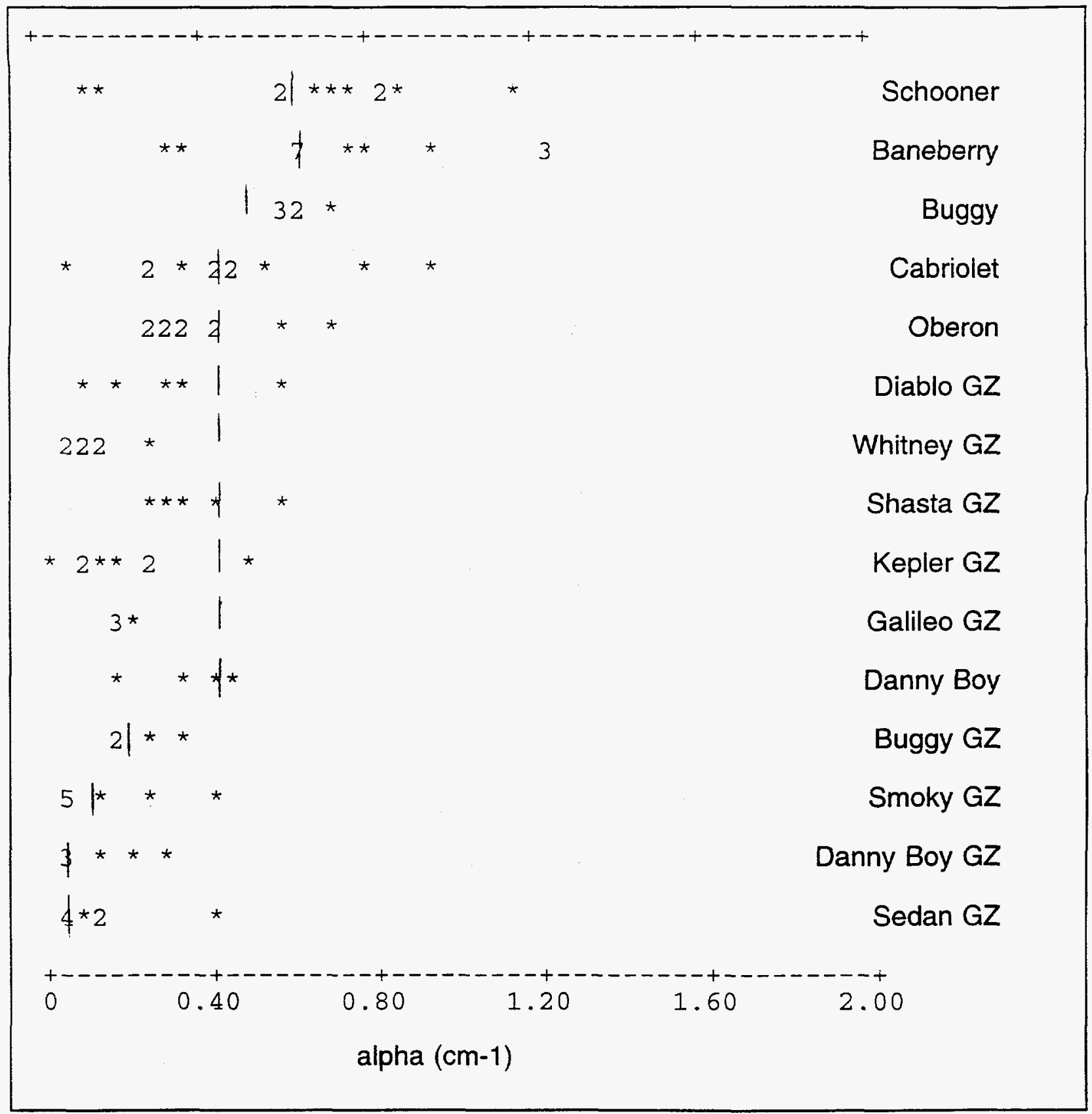

The vertical lines show the values used in analyzing the Radionuclide Inventory and Distribution Program spectra.

Figure 4-2

Calculated Values of the Inverse Relaxation Length for Cobalt-60. 


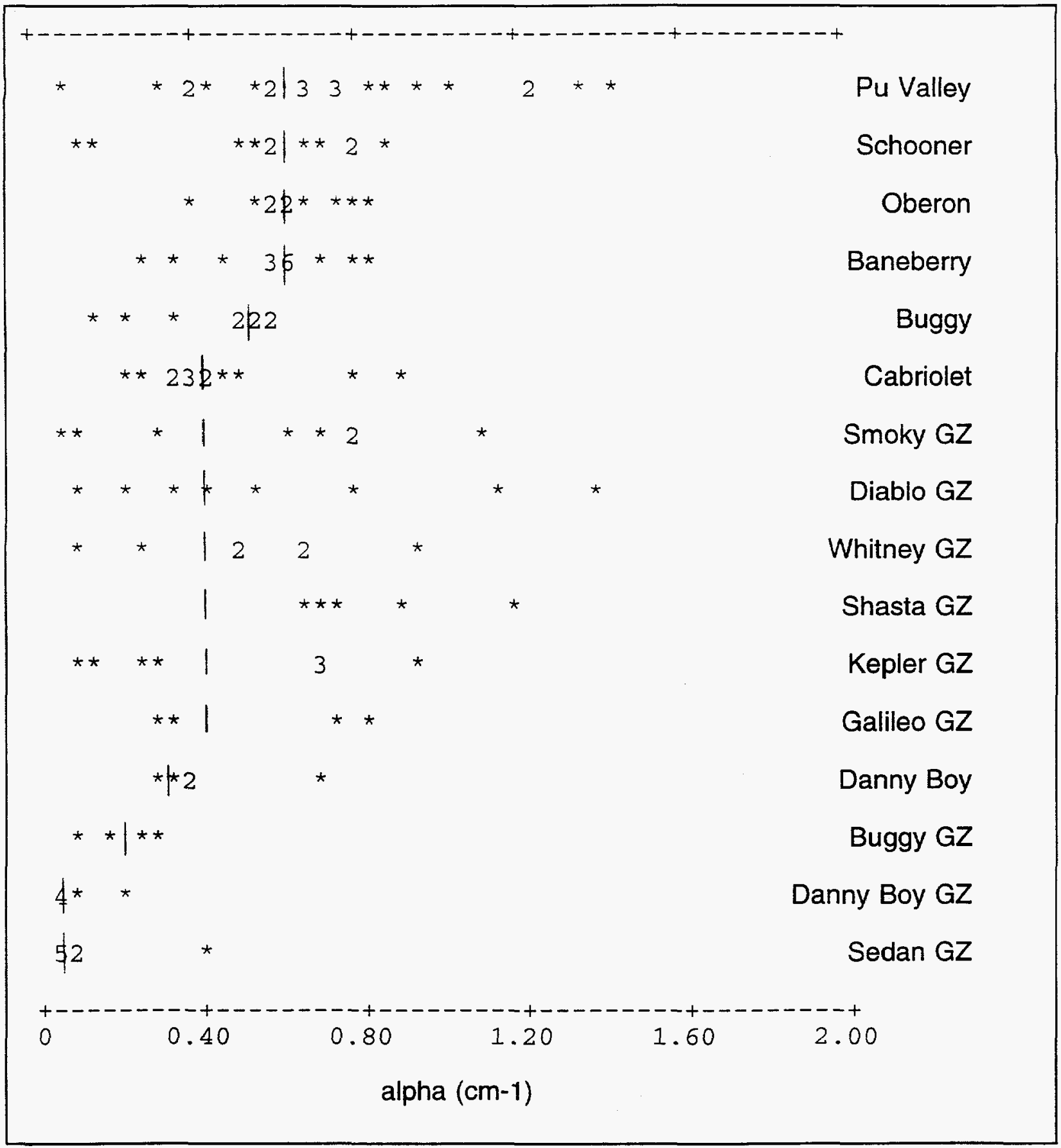

The vertical lines show the values used in analyzing the Radionuclide Inventory and Distribution Program spectra.

Figure 4-3

Calculated Values of the Inverse Relaxation Length for Cesium-137. 


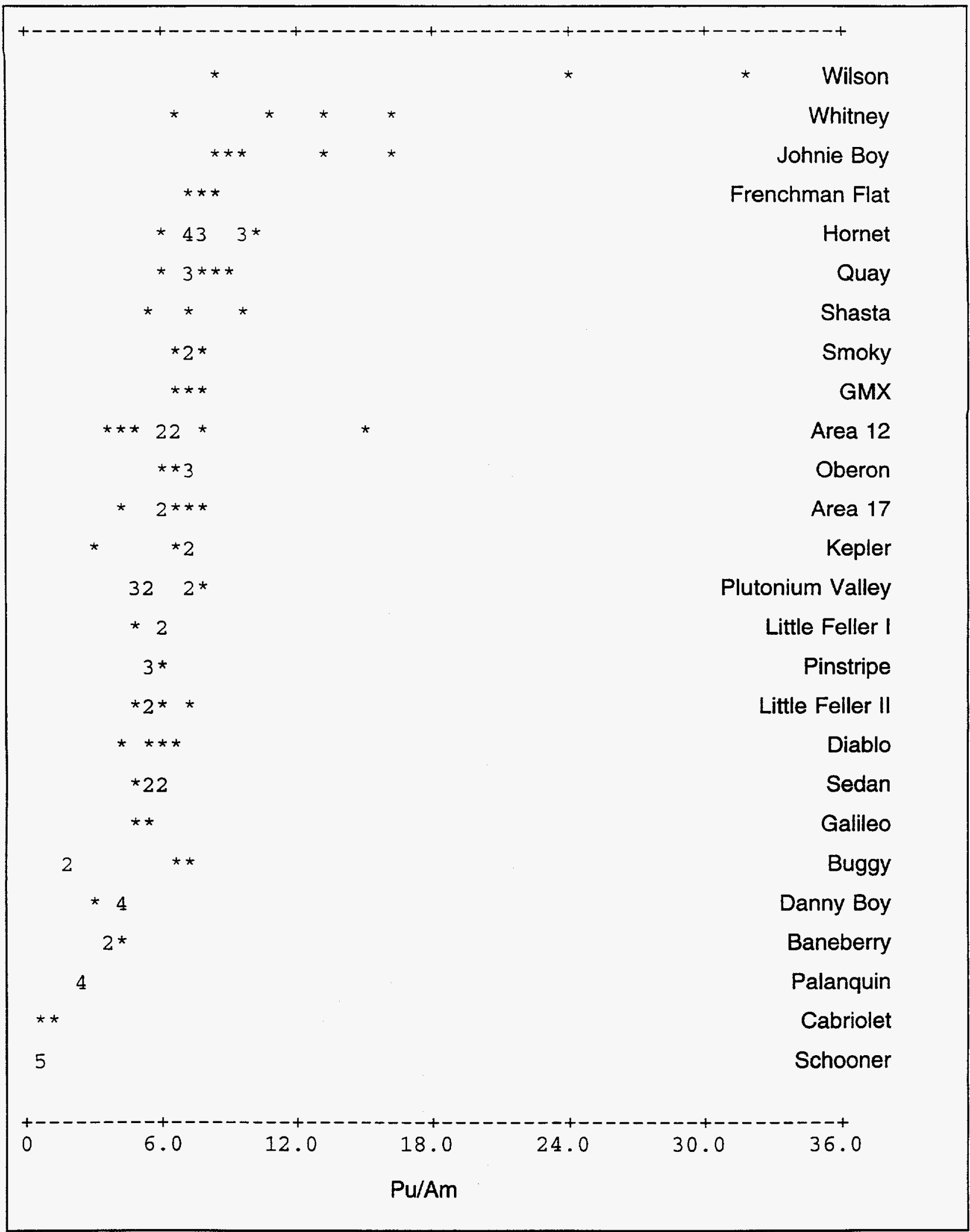

Figure 4-4

Ratios of Plutonium-239, -240 to Americium-241 in Radionuclide Inventory and Distribution Program Soil Samples 


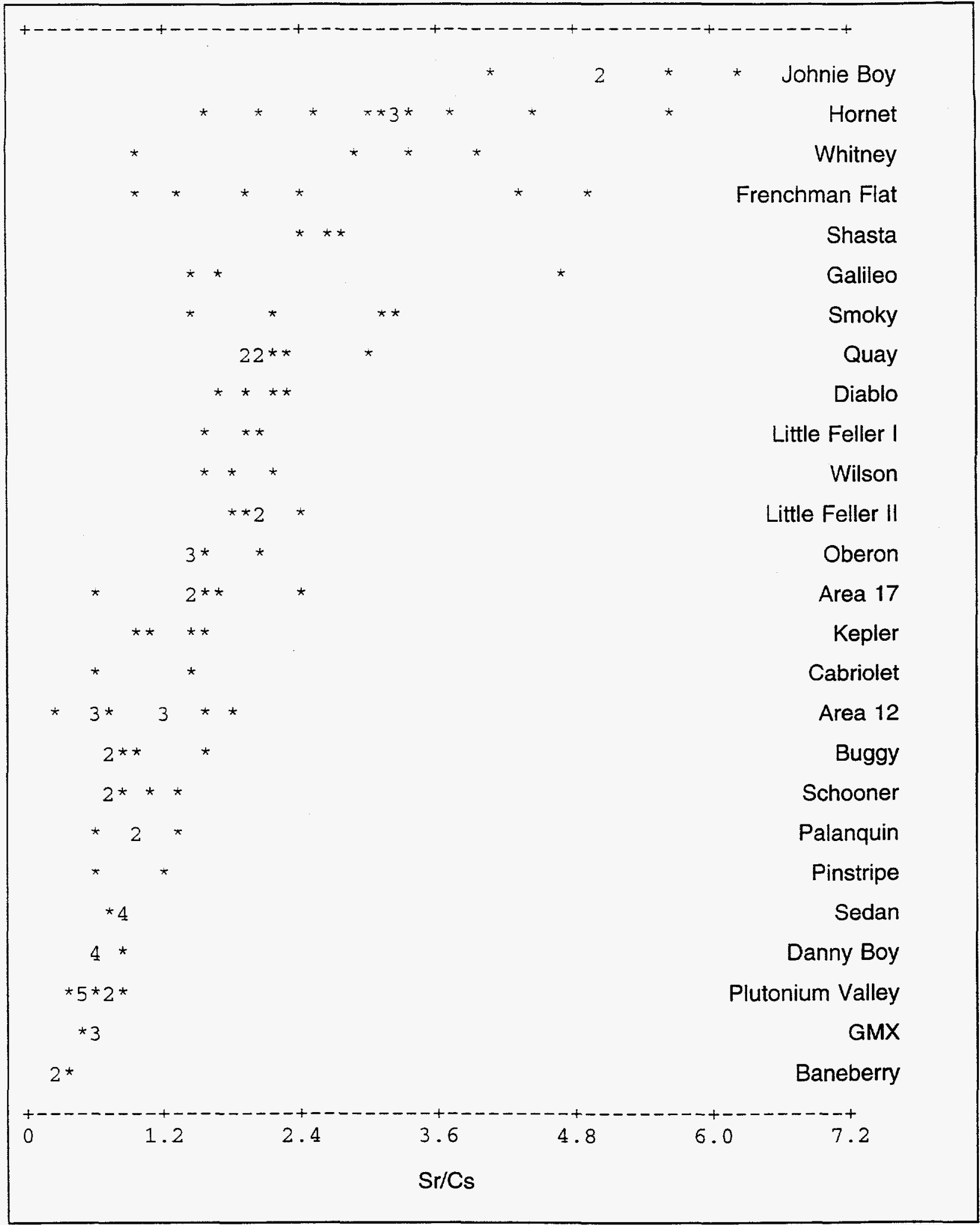

Figure 4-5

Ratios of Strontium-90 to Cesium-137 in Radionuclide Inventory and Distribution Program Soil Samples 
LANL and EAL Corporation for analysis. Those analyses suggested the possibility that material was being lost when the samples were dissolved at the REECo Laboratory, and REECo was reported to be examining its dissolution procedure (Essington and Mead, 1985).

A more formal QA program was in place for the rest of the RIDP soil analyses (about 1,400 samples). All aliquots of both field and QA samples were prepared and randomly assigned laboratory numbers under the supervision of the QA referee. The identity of the samples was not revealed to the analyst until the final results had been accepted. The labeled aliquot containers were photographed with the bulk samples to document the labeling. Also, duplicate aliquots of selected field and QA samples were routinely sent to other laboratories for analysis. About 160 duplicate aliquots and 90 aliquots of blank and reference soil were prepared and analyzed as part of the formal QA program.

In evaluating the QA data, a result was flagged if it differed from the mean of the replicates by more than three times its standard deviation. Flagged results were not automatically rejected, but were carefully evaluated before a decision was made. Relatively few results were flagged. Some of the discrepancies could be attributed to the hot particle problem, but many could not.

Overall, the QA results tended to confirm the accuracy of the spectrometric and radiochemical analysis of the soil samples. The occasional problems detected were considered unimportant because average values (with smaller uncertainties than individual values) were used in calculating ratios and inverse relaxation lengths, and because of the factor-of-two precision goal of the program.

\subsection{Availability of Data}

The RIDP collected data from every accessible region of the NTS where the aerial surveys showed levels of contamination above background, including all the GZs of aboveground tests and sites of underground tests where significant amounts of radioactivity reached the surface. In most of the RIDP surveys, the arrangement of measurement locations was a square grid of points. The spacing between locations was usually 400 or $500 \mathrm{ft}$, but was reduced to $250 \mathrm{ft}$ in some regions close to a GZ, and was sometimes increased to 1,000 or even $2,000 \mathrm{ft}$ to survey large areas with relatively low and evenly distributed contamination.

In the regions surveyed early in the program, measurements were not made at all the grid points. Instead, the measurements were concentrated in the regions where the level of 
radioactivity was changing most rapidly. This strategy was adopted to help better define radionuclide distributions, but it made estimating radionuclide inventories more complicated. In most areas, all of the grid points within a rectangular region were measured. This simplified the inventory estimation and reduced the amount of interpolation needed to map radionuclide distributions.

In some of the more remote regions of the NTS, the mountainous terrain made laying out a grid of measurement points impractical. Those regions were surveyed by making measurements along roads, jeep trails, and power lines.

A summary of the numbers of RIDP measurements is given in Table 4-2, which is a more accurate version of Table 2 in McArthur (1991). The total number of spectra recorded in the field (excluding calibration runs) is 3,976. The total number of in situ measurements given in the table is less because it does not include repeat measurements at some locations and measurements with questionable results that were not used in the inventory calculations. The number of locations for which valid RIDP data exist is, therefore, about 3,800. Soil profiles were collected from about 10 percent of those locations. Surface samples from about 40 percent of the profiles were analyzed chemically.

The original log sheets and tape cartridges for the in situ measurements are in the custody of EG\&G/EM. All the spectra, both calibration and field runs, have been archived at the DOE/NV computer center. The archived versions of the field spectra include the GAMANAL results. At present, DRI has copies of the log sheets and computer files containing the GAMANAL results from all the field spectra, but DRI is under no obligation to retain this material indefinitely. Copies of the data files were given to IT Corporation in 1993 . No "clean" version of the in situ data set (with repeat and questionable results deleted) currently exists.

Supplementary in situ measurements were made at about 55 locations at four sites where the terrain or high levels of contamination prevented vehicle access. Measurements near the Sedan crater and the Schooner and Buggy GZs were made with hand-held exposure rate meters; measurements in Plutonium Valley were made with a FIDLER. The results of these measurements have not been entered into computer data files.

The results of the RIDP soil analyses, including most of the QA results, were published in the first five RIDP reports. They are not known to exist in any computer data files. 
Table 4-2

Sample Sizes of Radionuclide Inventory and Distribution Programs Surveys

\begin{tabular}{|c|c|c|c|}
\hline Survey Region & $\begin{array}{c}\text { Number of } \\
\text { In Situ Measurement }\end{array}$ & $\begin{array}{l}\text { Number of } \\
\text { Soil Profiles }\end{array}$ & $\begin{array}{c}\text { Number of } \\
\text { Chemical Analyses }\end{array}$ \\
\hline Galileo & 136 & 11 & 3 \\
\hline Kepler & 122 & 9 & 4 \\
\hline Whitney & 173 & 21 & 9 \\
\hline Diablo & 53 & 8 & 2 \\
\hline Baneberry & 181 & 15 & 3 \\
\hline Sedan, Smoky & 262 & 25 & 9 \\
\hline Wilson & 207 & 23 & 3 \\
\hline Quay & 223 & 17 & 7 \\
\hline Hornet & 380 & 25 & 12 \\
\hline South Yucca & 46 & 0 & 0 \\
\hline Pahute Mesa & 25 & 0 & 0 \\
\hline Schooner & 61 & 11 & 5 \\
\hline Cabriolet & 219 & $13^{*}$ & 6 \\
\hline Johnnie Boy & 132 & 14 & 5 \\
\hline Little Feller i & 40 & 9 & 3 \\
\hline Little Feller II & 54 & $9^{*}$ & 5 \\
\hline Danny Boy & 107 & 11 & 5 \\
\hline Areas 25 and 26 & 181 & 24 & 5 \\
\hline Areas 17 and 18 & 176 & 15 & 6 \\
\hline Area 15 & 36 & 4 & 1 \\
\hline Pinstripe, GMX & 215 & 22 & 16 \\
\hline Plutonium Valley & 172 & 22 & 11 \\
\hline Buggy & 76 & 13 & 5 \\
\hline Areas 12 and 19 & 118 & 24 & 13 \\
\hline Oberon & 30 & 10 & 5 \\
\hline Frenchman Lake & 291 & 29 & 6 \\
\hline Miscellaneous & 81 & 15 & 8 \\
\hline Total & 3,798 & 399 & 157 \\
\hline
\end{tabular}

*Nevada Applied Ecology Group samples 


\subsection{Discussion of Historical Data Useability}

The overall objective of a remedial investigation and feasibility study or RCRA Facilities Investigation/Corrective Measures Study process is to determine the nature and extent of the threat posed by various substances and to evaluate proposed remedies. As a result, data in investigations of this type have a variety of uses. In addition to site characterization, data are used to assess potential hazards to worker health and safety, human health and ecological risk assessments, remedial selection. and remedial design.

The U.S. Environmental Protection Agency (EPA) has provided guidance on determining the quality of data that are required to satisfy the needs of various activities. Indicators of data quality are referred to as precision, accuracy, representativeness, completeness and comparability (PARCC) parameters. When evaluating site historical data to fulfill project needs, the PARCC parameters that were achieved must also be assessed.

The majority of data collected on the NTS fall into three categories: discrete samples analyzed in a laboratory; in situ gamma measurements using $\mathrm{NaI}(\mathrm{Tl})$ detection systems; and aerial gamma detection systems using $\mathrm{NaI}(\mathrm{Tl})$ crystals. The criteria used to determine data usability differ for each category and are discussed in the following sections.

\subsection{Discrete Soil Samples}

The evaluation of a chemical data base with respect to PARCC parameters and thus data useability is a more straightforward process than for radiological samples. Chemical methods are generally agency specified and use developed procedures with verifiable documentation of analytical procedures and results. Historical information on precision and accuracy for some methods, particularly the Contract Laboratory Program (CLP) parameters, are regularly updated and published.

EPA guidance is very specific on what analytical levels are appropriate for various data uses. Analytical techniques for chemical data are categorized with respect to the type of technology and degree of sophistication and documentation required and are referred to as analytical levels ranging from Level I to Level V. For example, Level I analytical support is generally field screening equipment, such as organic vapor meters that provide total vapor measurements, $\mathrm{pH}$, temperature, oxygen, and conductivity probes. These methods provide real-time analysis with minimal documentation and are not conducive to the generation of quantitative data. The uses of these types of data include worker health and safety issues and 
the identification of areas or samples for quantitative sampling. The resulting data are generally considered qualitative although certain measurements, such as $\mathrm{pH}$, when documented calibration with standard solutions is performed, result in qualitative data.

In contrast, Level IV analytical support is CLP Routine Analytical Services. These types of data are highly documented and contain information on initial and continuing calibration, GC/MS tuning, surrogate percent recovery, and matrix spike duplicates. Hard copies of reconstruction ion and GC chromatograms and of spectra for every sample, blank, and standard or spike run with a particular set of samples are also provided. The documentation is sufficient to allow for evaluation of data quality. Data of this caliber are used in risk assessments (although Level III data may be sufficient) and legal determinations because of the rigorous and verifiable $\mathrm{QA} /$ quality control $(\mathrm{QC})$ protocols and documentation.

Level $\mathrm{V}$ data include nonconventional parameters, such as radioisotopes, that may be used for toxicological and risk assessment purposes. NTS radiological samples analyzed in a laboratory may belong to analytical support category Level $\mathrm{V}$ if sufficient original documentation exists to assess the accuracy and precision of the method at the time the procedure was performed. If this documentation and sample custody, as it was practiced during that time period, could be established, these data could be sufficient for risk assessment purposes.

Several laboratories performed analyses including REECo. Documentation on QA/QC procedures performed on REECo samples is available either in hard copy or electronic format, depending on when the analyses were performed, and is archived at the CIC. Documentation for samples analyzed at McClellan Air Force Base still exists, but is very difficult to access due to problems associated with the transfer of hard copy data to microfilm and microfiche. QA/QC documentation for samples analyzed at Los Alamos, TMA/Norcal, LFE, and EAL no longer exists.

The opinions of Mr. E. Essington of Los Alamos National Laboratory and Mr. R. Osborne of McClellan Technical Operations Laboratory regarding the usability of the data indicate that the data were neither collected nor analyzed for risk assessment purposes. The samples were collected to provide supporting information for the calculation of plutonium inventory and do not have sufficient or documentable precision or accuracy for risk assessment purposes. 
In addition to analytical documentation, other criteria must be satisfied to establish the purposes for which the data may be used. The stringent criteria that must be satisfied for risk assessment purposes are very specific and are contained in several EPA documents (EPA, 1992a, 1992b, and 1987). Verification that samples were collected in accordance with a written sampling plan should exist. Sampling methods for NTS samples, still in use today, were developed as part of NAEG and RIDP. The methods have been documented in the literature and exist in archived files as memoranda and letters. However, no formal sampling plans were ever prepared. The sample collection logs exist in files at the NTS for many NAEG and RIDP sampling efforts although their extent is not known. The logs identify sample team members, sample locations, sample type, and date and time of collection. In addition, during RIDP, photographs were taken of sample locations; however, the repository for these photographs has not yet been located.

\subsection{In Situ Survey Data}

Data obtained from in situ surveys may be used to assess risk if they meet data usability requirements (EPA, 1992b). Just as with chemical data, the requirements include an assessment of the PARCCs parameters. In evaluating these parameters, detection and quantitation limits must be specified. These include the lower limit of detection that considers only instrument characteristics and the minimum detectable activity, both of which were reported in RIDP investigations.

As previously described, when determining the concentration of plutonium using in situ methods ${ }^{241} \mathrm{Am}$ is used as the surrogate analyte. In order to convert from americium activity per unit area to activity of plutonium per unit mass. a number of parameters must be known. If the ratio of americium to plutonium is known, the activity of plutonium can be calculated. In addition, the activity depth profile of Am within the soil, soil density, soil moisture content, and soil composition must be known to convert from activity over unit area to activity per unit weight. The major sources of error in the calculated nuclide concentrations are the depth profile and the concentration ratio. The other factors also introduce errors, but to a much lesser extent.

As shown in Chapter 4 , the reported ratios for ${ }^{239 / 240} \mathrm{Pu}$ to ${ }^{241} \mathrm{Am}$ vary within a single shot area. The ratio at the safety shot areas, Plutonium Valley and GMX, is reported to lie between approximately five and eight. This variation can introduce an error of a factor of nearly 2 in the final plutonium calculation. At the locations of atmospheric events, the ratio range is generally wider, which produces an even larger error. To minimize the error, an 
average value was assumed for each of the individual sites, and all data were converted using the average value.

The errors introduced by uncertainty in the depth distribution of ${ }^{241}$ Am are similarly large. The measured values of alpha at Plutonium Valley range from approximately 0.03 to 1.2. This array of values results in a factor of three difference in surface concentrations. Again, in RIDP studies, an average value was assumed and applied to all ${ }^{241} \mathrm{Am}$ flux measurements.

While assumptions of this type may be adequate for estimating inventory and distribution, they are unsuitable for a quantitative assessment of risk. In order to "upgrade" the data, better definition of depth distribution and radionuclide ratios must be obtained to minimize the uncertainty.

\subsection{Aerial Survey Data}

The aerial survey data, as currently collected, should be used only as a screening tool to locate areas of elevated land surface activity. Data are presently collected by multiple passes along a site grid by a helicopter at an altitude of approximately $31 \mathrm{~m}(100 \mathrm{ft})$ and a ground speed of approximately 70 knots $(80 \mathrm{mph})$. The helicopter uses a $\mathrm{NaI}(\mathrm{Tl})$ detector, and corrections are made for cosmic interference and other factors. The data are collected continuously and recorded at one-second intervals along the flight grid, which at the stated ground speed yields a 37-m (118-ft) long data interval (Proctor and Hendricks, 1994). An average value is assumed for both radionuclide depth distribution and ratios.

There are various ways to process the resulting data. One example, shown in Figure 5-1, is the result of a December 1993 flyover of the Double Tracks site on the NAFR near Goldfield, Nevada, to determine plutonium levels in soil. To improve sensitivity, the spectral data were summed within a $140-\mathrm{m}$ by $140-\mathrm{m}$ ( $450-\mathrm{ft}$ by $450-\mathrm{ft}$ ) area (pixel), and the spectral value was assumed to lie within the center of the pixel. The results were then contoured. Superimposed on the flyover data are the results of NAEG soil samples collected in 1978. Overall agreement is quite good with the exception of the skewing of the flyover contours particularly around $G Z$ to the west. The skewing is a result of the processing routine selected to improve sensitivity. Other processing routines may provide better spatial resolution, but may decrease sensitivity. An example of another processing routine is shown in Figure 5-2. 


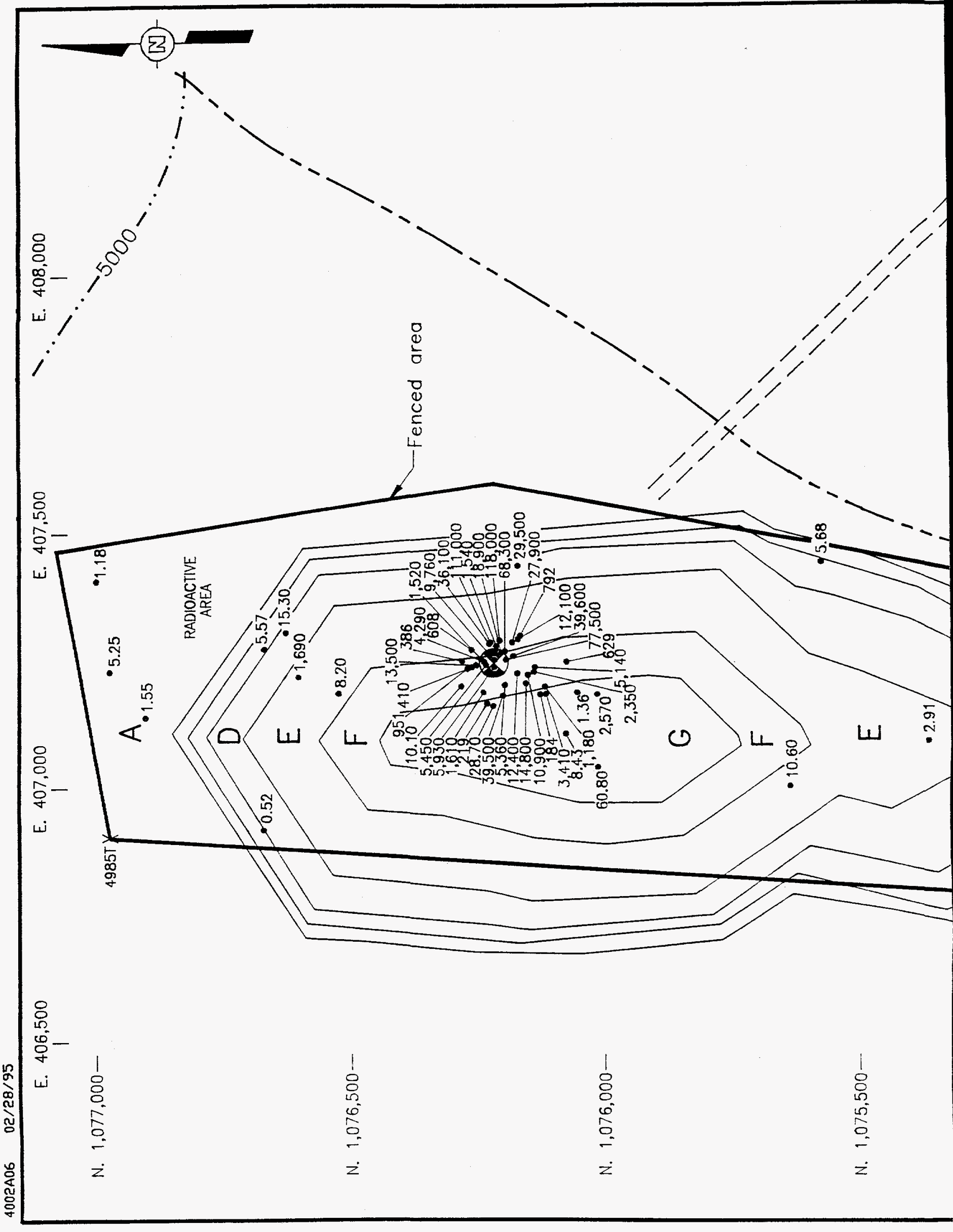


N. $1,074,500-$
SCALE

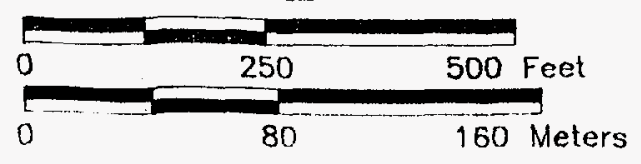

\begin{tabular}{|c|c|}
\hline $\begin{array}{c}\text { LETTER } \\
\text { LABEL }\end{array}$ & $\begin{array}{c}\text { PLUTONIUM } \\
\text { pCi/gram }\end{array}$ \\
\hline A & $<25$ \\
B & $25-30$ \\
C & $30-40$ \\
D & $40-60$ \\
E & $60-100$ \\
F & $100-130$ \\
G & $130-200$ \\
\hline
\end{tabular}

Doto were taken at 100 feet-above ground of 70 knots ground speed. The detector array consisted of eight-2 $244 \times 16$-inch Nol(Ti) detectors. A $450-x \quad 450$-foot pixel wos used for summing dato.

\section{Conversion ossumptions were:}

cosine detector response

3-cm reloxotion depth

2.5-cm somple depth

$\mathrm{Pu} / \mathrm{Am}$ rotio $=10$

Photopeok cts $=$ window $\mathrm{cts} \times 1.6$

\section{LEGEND}

$$
= \pm=\operatorname{Road}
$$

- - Drainage

Ground zero

- Discrete radiochemical samples collected as part of Nevada Applied Ecology Group

study. Results reported in $\mathrm{pCi} / \mathrm{g}$.
N. $1,074,000-$

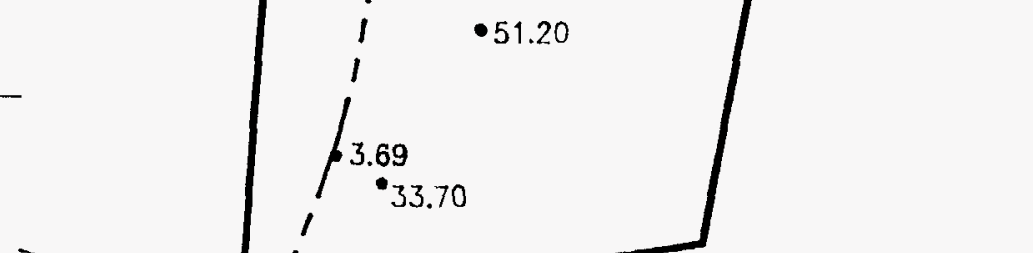

$==\frac{\text { FIGURE }}{5-1}$

AERIAL AND

RADIOCHEMICAL PLUTONIUM

SAMPLING LOCATIONS

DOUBLE TRACKS, NELLIS AIR FORCE RANGE 


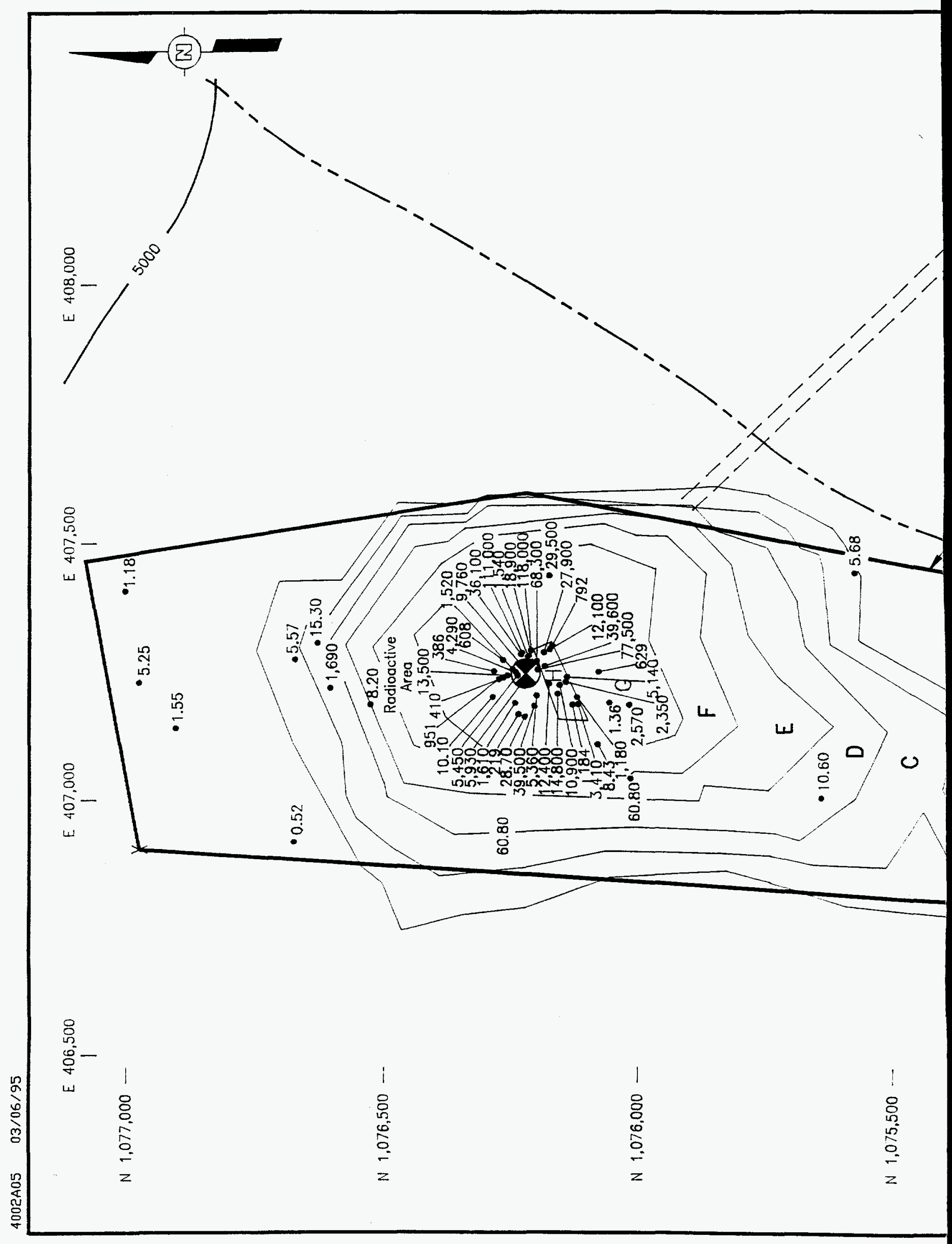




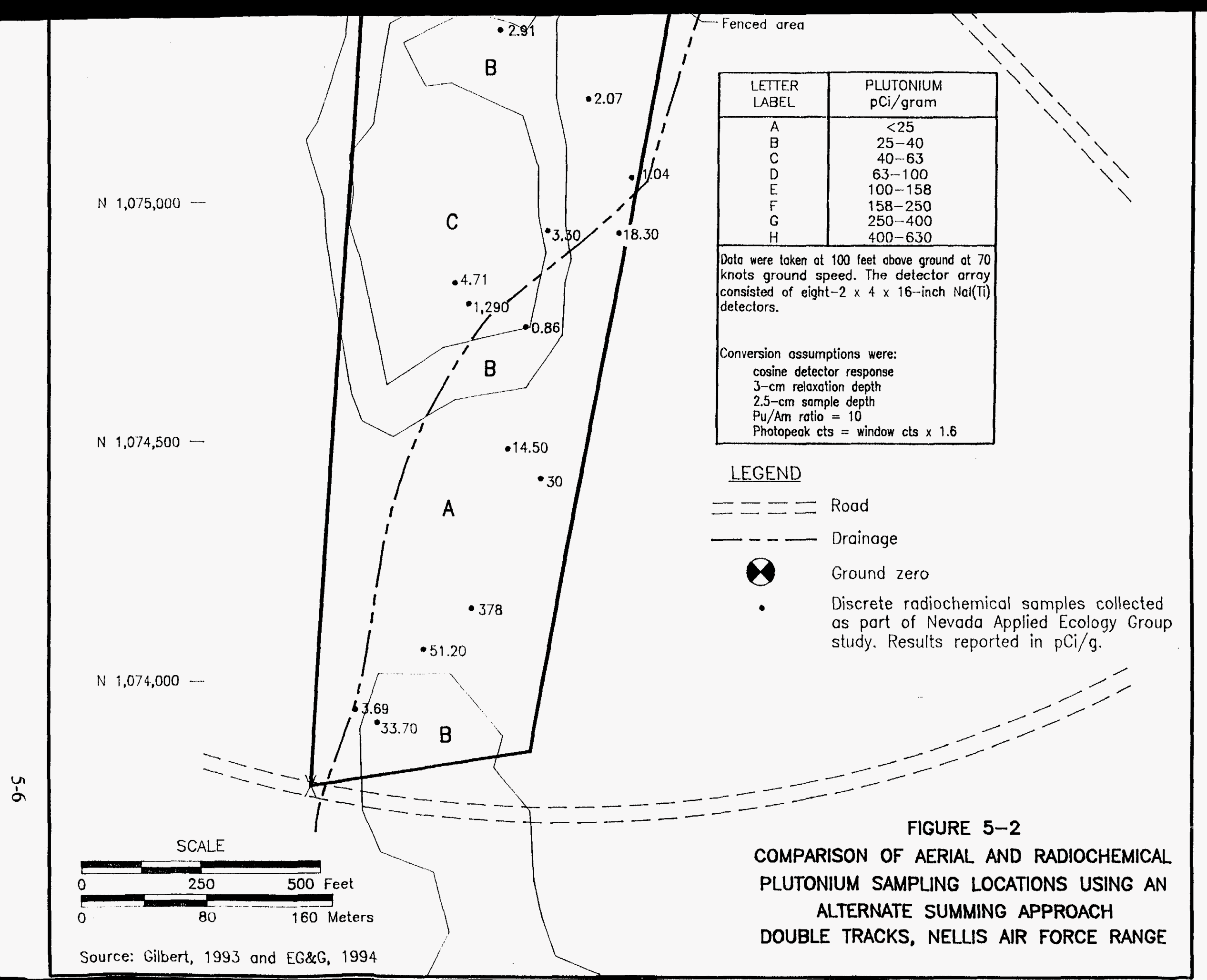


Because of the high level of uncertainty associated with this method in its present form, the results should be used as a screening tool. The precision and accuracy are not adequate for characterization or risk assessment purposes.

\subsection{Summary}

The results of this preliminary data review indicate that while much data have been collected over the years at the NTS and other associated locations, very little of the data has the stringent QA/QC pedigree that is required for use in a quantitative risk assessment under CERCLA. It would be very difficult, if not impossible, to show that the required PARCCs parameters were met. While the data were collected using state-of-the-art techniques and analytical methods for the time, they were collected for the purposes of estimating inventory and distribution to within no more than a factor of two. The data were never intended for assessing human health effects.

Most of the data are useable for screening purposes and can be made usable for characterization purposes with limited additional field studies. The quality of the in situ data can be improved with a better understanding of the vertical distribution of plutonium in the upper soil column and the ratios of various radionuclides. 


\subsection{References}

Anspaugh, L.R. and J.F. Kordas, Lawrence Livermore Laboratory, 1980. Assessment of the Nevada Test Site Inventory and Distribution Project, UCRL-52967, Livermore, CA.

Anspaugh, L.R., 1976, "In situ methods for quantifying specific radionuclides," IEEE trans. on Nuclear Science, NS-23:1190-1196.

Barnes, M.G., J.J. Giacomini, R.T. Reiman, and B. Elliot, Desert Research Institute, 1980, NTS Radiological Assessment Project: Results for Frenchman Lake Region of Area 5 , DOE/DP01253-17, Reno, NV.

Beck, H.L., J. Decampo, and C. Gogolak, U.S. Atomic Energy Commission, 1972, In Situ Ge (Li) and $\mathrm{Na}$ (T1) Gamma-Ray Spectrometry, HASL-258, New York, NY.

Bluitt, C.M., EG\&G Energy Measurements, Inc.. 1986. An Aerial Radiological Survey of Areas 16 and 30, Nevada Test Site, EGG-10282-1118, Las Vegas, NV.

Brady, D.N. and B.W. Church. U.S. Department of Energy, 1975, Discussion of NAEG Distribution and Inventory Program sampling Data in Preparation for Initiation Phase III, NVO-153, Las Vegas, NV.

Brady, D.N., L.M. Rakow, and C.E. Rosenberry, U.S. Department of Energy, 1978, On-Site REECo Support Activities for the Nevada Applied Ecology Group, Las Vegas, NV.

Britton, G.L. and E.R. Sorom, Reynolds Electrical \& Engineering Co., Inc., 1981, Memorandum, Soil Storage in Bldg. 2106, Las Vegas, NV.

Church, B.W., D.N. Brady, I. Aoki, and W.A. Bliss, U.S. Department of Energy, 1974, Distribution and Inventory Element Activities On-NTS and Off-NTS, NVO-142,

Las Vegas, NV.

Clark, H.W., EG\&G Energy Measurements, Inc., 1983, An Aerial Radiological Survey of Area 11, Nevada Test Site, EGG-10282-1004, Las Vegas, NV.

EG\&G, 1994, Aerial Radiological Survey of the Double Track Site, NAFR 71, Pu Isopleth, Las Vegas, NV.

Essington, E.H., Los Alamos National Laboratory, 1994, Draft Philosophy and Design of a Quality Assurance Program for the Nevada Applied Ecology Group, Los Alamos, NM.

Essington, E.H., 1989, "Reliability of Soil Analysis," Appendix C, in R.D. McArthur and S.W. Mead, Desert Research Institute, Nevada Test Site Radionuclide Inventory and Distribution Program: Report \#5. Areas 5, 11, 12, 15, 17, 18, 19, 25, 26, and 30. DOE/NV/10384-26, Publication No. 45067, Las Vegas, NV. 
Essington, E.H., U.S. Department of Energy, 1987a, Soil Investigations for the NAEG: A Historical Review and Current Status, NVO-272. Las Vegas, NV.

Essington. E.H., U.S. Department of Energy, 1987b, Soil Radionuclide Distribution Studies for the Nevada Applied Ecology Group -- 1981, NVO-272, Las Vegas, NV.

Essington, E.H. and S.W. Mead, 1985, "Quality Assurance for Measurements of Soil Radioactivity," Appendix C, in R. D. McArthur and J.F. Kordas, Nevada Test Site Radionuclide Inventory and Distribution Program: Report \#2, Areas 2 and 4.

DOE/NV/10162-20, Las Vegas, NV.

Essington, E.H., U.S. Department of Energy, 1978, Soil Radioactivity Distribution Studies for the Nevada Applied Ecology Group, NVO-192, Las Vegas, NV.

Feimster, E.L., EG\&G Energy Measurements, Inc., 1985, An Aerial Radiological Survey of Areas 18 and 20, Nevada Test Site, EGG-10282-1093, Las Vegas, NV.

Friesen, H.N., U.S. Department of Energy, 1992. Summary of the Nevada Applied Ecology Group and Correlative Programs, DOE/NV-357, Las Vegas, NV.

Fritzsche, A.E., EG\&G Energy Measurements, Inc., 1982, An Aerial Radiological Survey of Areas, 1, 2, 3, 4, 6, 7, 8, 9, 10, 12, 15 and 17, Yucca Flat, Nevada Test Site, EGG-1183-1808, Las Vegas, NV.

Fritzsche, A.E., EG\&G Energy Measurements, Inc., 1979, An Aerial Radiological Survey of Area 13, Nevada Test Site, EGG-1183-1752, Las Vegas, NV.

Gilbert, R.O., 1993, Personal Communication, NAEG Soil Sample Data.

Gilbert, R.O., J.C. Simpson, D.W. Engel, and R.R. Kinnison, U.S. Department of Energy, 1987, Estimating Isotopic Ratios, Spatial Distribution, and Inventory of Radionuclides at Nuclear Sites 201, 219 and 221. NVO-272, Las Vegas, NV.

Gilbert, R.O., E.H. Essington, D.N. Brady, P.G. Doctor, and L.L. Eberhardt, U.S. Department of Energy, 1977, Statistical Activities During 1976 and the Design and Initial Analysis of Nuclear Site Studies, NVO-181, Las Vegas, NV.

Gilbert, R.O. and L.L. Eberhardt, U.S. Department of Energy, 1976, Statistical Analysis of "A Site" Data and Interlaboratory Comparisons for the Nevada Applied Ecology Group, NVO-159, Las Vegas, NV.

Gilbert R.O., L.L. Eberhardt, E.B. Fowler, E.M. Romney, E.H. Essington, and J.E. Kinnear, U.S. Department of Energy, 1975, Statistical Analysis of Pu-239/240 and Am-241 Contamination of Soil and Vegetation on NAEG Study Sites, NVO-153, Las Vegas, NV. 
Gilbert R.O., L.L. Eberhardt. E.B. Fowler, and E.H. Essington. 1974, Statistical Design Aspects of Sampling Soil for Plutonium, Energy Research and Development Administration (ERDA) Symposium Series 38.

Gunnink. R. and J.B. Niday, Lawrence Livermore National Laboratory, 1971, Computerized Quantitative Analysis by Gamma-ray Spectrometry, Vol. 1-5, UCRL-51061, Livermore, CA.

Hopkins, R.C., EG\&G Energy Measurements, Inc., 1992, Off-Site Plutonium Plume Survey, Nevada Test Site, NV-93-090, Las Vegas, NV.

Jobst, J.E., EG\&G Energy Measurements, Inc., 1986, An Aerial Radiological Survey of Areas 12, 15, 17, and 19, Nevada Test Site, EGG-10282-1113, Las Vegas, NV.

Jobst, J.E., EG\&G Energy Measurements, Inc., 1979, An Aerial Radiological Survey of Clean Slates 1, 2 and 3, and Double Track: Tonopah Test Range, Central Nevada, EGG-1183-1737, Las Vegas, NV.

Kayuha, H.J, I. Aoki, and D.L. Wireman, U.S. Department of Energy, 1974, REECo Field Activities and Sample Logistics in Support of the Nevada Applied Ecology Group, NVO-142, Las Vegas, NV.

Kirby, J.A., L.R. Anspaugh, P.L. Phelps, and G.W. Huckabay, 1977, "A comparison of in situ gamma soil analysis and soil sampling data for mapping ${ }^{241} \mathrm{Am}$ and ${ }^{239} \mathrm{Pu}$ soil concentrations at the Nevada Test Site," IEEE Trans. on Nuclear Science, NS-24:587-590.

Kordas, J.F. and L.R. Anspaugh, Lawrence Livermore National Laboratory, 1982, Nevada Test Site Radionuclide Inventory and Distribution Project Operations Plan, UCID-19413, Livermore, CA.

Lee, K.D., REECo, 1994, Personal Communication.

Markwell, F.R. and B.W. Church, U.S. Department of Energy, 1977, Nevada Applied Ecology Group Distribution and Inventory Sampling Data, NVO-171, Las Vegas, NV., p 233.

McArthur, R.D., Desert Research Institute, 1991, Radionuclides in Surface Soil at the Nevada Test Site, DOE/NV/10845-02, Publication No. 45077, Las Vegas, NV.

McArthur, R.D. and S.W. Mead, Desert Research Institute, 1989, Nevada Test Site Radionuclide Inventory and Distribution Program: Report \#5. Areas 5, 11, 12, 15, 17, 18, 19, 25, 26, and 30, DOE/NV/10384-26, Publication No. 45067, Las Vegas, NV.

McArthur, R.D. and S.W. Mead. Desert Research Institute, 1988, Nevada Test Site Radionuclide Inventory and Distribution Program: Report \#4. Areas 18 and 20 , DOE/NV/10384-22. Publication No. 45063, Las Vegas, NV. 
McArthur, R.D. and S.W. Mead, Desert Research Institute, 1987, Nevada Test Site Radionuclide Inventory and Distribution Program: Report \#3. Areas 3, 7, 8, 9, and 10, DOE/NV/10384-15, Publication No. 45056, Las Vegas, NV.

McArthur, R.D. and J.F. Kordas, Desert Research Institute, 1985, Nevada Test Site Radionuclide Inventory and Distribution Program: Report \#2. Areas 2 and 4, DOE/NV/10162-20, Publication No. 45-41, Las Vegas, NV.

McArthur, R.D. and J.F. Kordas, Desert Research Institute, 1983, Radionuclide Inventory and Distribution Program: The Galileo Area, DOE/NV/10162-14, Publication No. 45035, Las Vegas, NV.

McArthur, R.D. and S.W. Mead, Desert Research Institute, 1988, Nevada Test Site Radionuclide Inventory and Distribution Program: Report \#4. Areas 18 and 20, DOE/NV/10384-22, Publication No. 45063, Las Vegas, NV.

Patton, K., REECo, 1994, Personal Communication, Soil Sampling Techniques Used for NAEG.

Proctor, A.E. and T.J. Hendricks, EG\&G Energy Measurements, Inc., 1994, An Aerial Radiological Survey of the Tonopah Test Range Including Clean Slates 1, 2, 3, Roller Coaster, Decontamination Area, Cactus Springs Ranch, Target Areas, Central Nevada, EGG-11265-1086, Las Vegas, NV.

REECo, 1977, Security Plan for Processing NAEG Sample Data from NTS Nuclear Event Sites, Las Vegas, NV.

Rosenberry, C., REECo, 1994, Personal Communication.

Tipton, N.J., EG\&G Energy Measurements, Inc., 1979, An Aerial Radiological Survey of Areas 25 and 26, Nevada Test Site, EGG-1183-1745, Las Vegas, NV.

U.S. Department of Energy, 1987, NVO-272, The Dynamics of Transuranics and Other Radionuclides in Natural Environments, W.A. Howard, R.G. Fuller, Eds.

U.S. Department of Energy, 1985, NVO-224, The Radioecology of Transuranics and Other Radionuclides in Desert Ecosystems, W.A. Howard, P.B. Dunaway, and R.G. Fuller, Eds.

U.S. Department of Energy, 1976, NVO-159, Studies of Environmental Plutonium and Other Transuranics in Desert Ecosystems, M.G. White and P.B. Dunaway, Eds.

U.S. Department of Energy, 1975, NVO-153, The Radioecology of Plutonium and Other Transuranics in Desert Environments, M.G. White and P.B. Dunaway, Eds.

U.S. Department of Energy, 1974, NVO-142, The Dynamics of Plutonium in Desert Environments, P.B. Dunaway and M.G. White, Eds. 
U.S. Environmental Protection Agency, 1992a, Guidance for Data Useability in Risk Assessment (Part A) Final, Publication 9285.7-09A, Washington, DC.

U.S. Environmental Protection Agency, 1992b, Guidance for Data Useability in Risk Assessment (Part B) Final, Publication 9285.7-09B/PB92-963362, Washington, DC.

U.S. Environmental Protection Agency, 1987. Data Quality Objectives for Remedial Response Activities Development Process, Washington, DC.

Wireman. D.L. and H.J. Kayuha, U.S. Department of Energy, 1976, Standard NAEG Procedures for Preparation of Soil Samples from NAEG Intensive Study Areas, NVO-166, Volume 1, Las Vegas, NV. 


\section{Distribution List}

\section{$\underline{\text { Copies }}$}

Wayne Bliss, Manager

Reynoids Electrical \& Engineering Co., Inc.

MS428

P. O. Box 98521

Las Vegas, NV 89193-8521

IT Corporation

4330 S. Valley View, Suite 114

Las Vegas, NV 89103

Roger Jacobson, Manager

Desert Research Institute

MS505

P. O. Box 19040

Las Vegas, NV 89132-0040

U.S. Department of Energy

Office of Scientific and Technical Information 175 Oak Ridge Turnpike

P. O. Box 62

Oak Ridge, TN 37831

Leo Rogers, Manager

EG\&G Measurements, Inc.

MS570/RSL-11

P. O. Box 1912

Las Vegas, NV 89125

Monica Sanchez, Project Manager

U.S. Department of Energy

MS505

P. O. Box 98518

Las Vegas, NV 89193-8518 